IFIC/02-31

FTUV/02-0719

\title{
QCD moment sum rules for Coulomb systems: the charm and bottom quark masses
}

\author{
Markus Eidemüller \\ Departament de Física Teòrica, IFIC, Universitat de València - CSIC, \\ Apt. Correus 22085, E-46071 València, Spain
}

\begin{abstract}
In this work the charm and bottom quark masses are determined from QCD moment sum rules for the charmonium and upsilon systems. To illustrate the special character of these sum rules when applied to Coulomb systems we first set up and study the behaviour of the sum rules in quantum mechanics. In our analysis we include both the results from nonrelativistic QCD and perturbation theory at next-nextto-leading order. The moments are evaluated at different values of $q^{2}$ which correspond to different relative influence among the theoretical contributions.

In the numerical analysis we obtain the masses by choosing central values for all input parameters. The error is estimated from a variation of these parameters. First, the analysis is performed in the pole mass scheme. Second, we employ the potential-subtracted mass in intermediate steps of the calculation to then infer the quark masses in the $\overline{\mathrm{MS}}$-scheme. Our final results for the pole- and $\overline{\mathrm{MS}}$ masses are: $M_{c}=1.75 \pm 0.15 \mathrm{GeV}, m_{c}\left(m_{c}\right)=1.19 \pm 0.11 \mathrm{GeV}$, $M_{b}=4.98 \pm 0.125 \mathrm{GeV}$ and $m_{b}\left(m_{b}\right)=4.24 \pm 0.10 \mathrm{GeV}$.
\end{abstract}

Keywords: Quarkonia, Quark masses, QCD sum rules PACS: 12.15.Ff, 12.38.Lg, 14.65.Dw, 14.65.Fy 


\section{Introduction}

Quantum Chromodynamics, the fundamental theory of strong interactions, represents a basic building stone of the Standard Model. The determination of its parameters remains an essential task within modern particle physics. The strong coupling constant can be obtained from many different sources to rather high accuracy [1]. Investigations for the quark masses face much more severe problems. Confinement effects must be taken into account for most systems sensitive to the masses. Therefore, apart from the top mass, nonperturbative methods like QCD sum rules [2, 3, 4], lattice QCD [5, 6] or chiral perturbation theory [7,8] have to be employed.

The extraction of the heavy quark masses was among the first applications of the method of QCD sum rules [2,3]. In this framework the hadronic parameters can be related to a perturbative QCD calculation including the nonperturbative condensate contributions. The analyses were later updated and extended [9, 10]. Recent times have seen a renewed interest in these investigations. With the development of nonrelativistic QCD (NRQCD) [1], 12 it has been recognised that the Coulombic form of the potential plays a major role in determinations of the charm and bottom quark masses. The contributions from NRQCD have been calculated up to next-next-to-leading order (NNLO) [13, 14, 15. In a region where the system is sensitive to mass effects, they dominate the theoretical evaluation of the sum rules over a pure perturbative expansion in the strong coupling constant.

The fundamental quantity in this type of sum rule analysis is the vacuum polarisation function $\Pi\left(q^{2}\right)$ :

$$
\Pi_{\mu \nu}\left(q^{2}\right)=i \int d^{4} x e^{i q x}\left\langle T\left\{j_{\mu}(x) j_{\nu}^{\dagger}(0)\right\}\right\rangle=\left(q_{\mu} q_{\nu}-g_{\mu \nu} q^{2}\right) \Pi\left(q^{2}\right),
$$

where the relevant vector current is represented either by the charm $j_{\mu}^{c}(x)=$ $\left(\bar{c} \gamma_{\mu} c\right)(x)$ or the bottom current $j_{\mu}^{b}(x)=\left(\bar{b} \gamma_{\mu} b\right)(x)$. Via the optical theorem, the experimental cross section $\sigma\left(e^{+} e^{-} \rightarrow c \bar{c}, b \bar{b}\right)$ is related to the imaginary part of $\Pi(s)$ :

$$
R_{c, b}(s)=\frac{1}{Q_{c, b}^{2}} \frac{\sigma\left(e^{+} e^{-} \rightarrow c \bar{c}, b \bar{b}\right)}{\sigma\left(e^{+} e^{-} \rightarrow \mu^{+} \mu^{-}\right)}=12 \pi \operatorname{Im} \Pi_{c, b}(s+i \epsilon) .
$$

Usually, moments of the vacuum polarisation are defined by taking derivatives of the correlator at $s=0$. However, in this work we will allow for an arbitrary evaluation point $s=-4 m^{2} \xi$ to define the dimensionless moments:

$$
\mathcal{M}_{n}(\xi)=\left.\frac{12 \pi^{2}}{n !}\left(4 m^{2} \frac{d}{d s}\right)^{n} \Pi(s)\right|_{s=-4 m^{2} \xi} .
$$


As we will see in more detail later, the parameter $\xi$ encodes much information about the system. By taking $\xi$ larger the evaluation point moves further away from the threshold region. As already discussed in [3], this leads to a better convergence for the perturbative expansion. The price to be paid is a small dependence of the moments on the mass. This again limits the possible accuracy when extracting the mass from the moments. Using a dispersion relation we can write the moments $\mathcal{M}_{n}(\xi)$ as an integral over the spectral density $R(s)$ :

$$
\mathcal{M}_{n}(\xi)=\left(4 m^{2}\right)^{n} \int_{s_{\min }}^{\infty} d s \frac{R(s)}{\left(s+4 m^{2} \xi\right)^{n+1}}=2 \int_{0}^{1} d v \frac{v\left(1-v^{2}\right)^{n-1} R(v)}{\left(1+\xi\left(1-v^{2}\right)\right)^{n+1}}
$$

The last equation represents a convenient way to express the moments through the velocity of the heavy quarks $v=\sqrt{1-4 m^{2} / s}$.

The moments can either be calculated theoretically, including Coulomb resummation, perturbation theory and nonperturbative contributions, or be obtained from experiment. In this way one can relate the heavy quark masses to the hadronic properties of the quark-antiquark systems. The theoretical setup is identical for the charmonium and upsilon and in the first chapters we will thus not specify the quark content. The difference will become crucial for the phenomenological part and the numerical analysis. First we will perform the analysis for the bottom quark mass where the theoretical expansions converge better and then determine the charm quark mass from the more delicate charmonium system.

Of decisive importance for the determination of the masses is the threshold behaviour. Here the system reacts very sensitive to mass effects. A small change in the mass leads to a relatively large variation of the moments. Thus the mass can in principle be determined with rather high accuracy. Therefore we try to develop a consistent physical description of the threshold region which includes all theoretical contributions and perform a matching between the low and high energy region.

Before turning to a discussion of the individual contributions, the next section shall highlight the special character of the sum rules when applied to the Coulomb system. To this end, we present the sum rules in the framework of quantum mechanics which were developed in [16]. Then we present here for the first time the application of the moment sum rules to a system governed by the Coulomb potential. All theoretical contributions can be described analytically. The sum rules show the dependence of the mass on the moments explicitly. Furthermore, the dependence of the pole and continuum parts on $n$ and $\xi$ will be studied and we investigate how these parameters determine the relative influence between the different contributions. 
The results from NRQCD can be described similar to the quantum mechanical sum rules. They contain a nonrelativistic Green's function which is composed of a continuous spectrum above and poles below threshold. We will isolate these contributions and analyse their influence on the mass and the error separately. Namely the poles will give the largest individual contribution to the mass. Therefore we will not use the nonrelativistic expansion to the energy levels itself which suffers from large corrections, but rather evaluate directly the Green's function in a region where the expansion is expected to work.

As already done in our previous work for the charmonium system [17, in the theoretical description of the correlator we will use information both from Coulomb resummation and perturbation theory. The perturbative expansion contains a part which is not included in NRQCD and will be added to the correlator. The nonrelativistic spectral density is valid for low velocities whereas the perturbative spectral density is well suited for high velocities. Therefore we will introduce a separation velocity to construct a spectral density for the full energy range. This will allow us to obtain a stable mass prediction for a wide range of parameters. The matching will turn out to be very smooth for the upsilon system but leading already to a gap for the charmonium. The analysis will show, however, that this mismatch has no numerical influence on the mass. The parameter $\xi$ will be used to shift the analysis towards a more perturbative region. There the expansions of NRQCD and perturbation theory converge faster and the different theoretical contributions are more equally distributed. This will reduce the systematic uncertainty of the sum rule approach. Since the sum rules keep the analytic dependence on the input parameters, we will investigate in detail their influence on the mass. To estimate the error we will vary each of the parameters in suitably large chosen windows. The dominant uncertainty will come from the nonrelativistic expansion and manifests itself in a large dependence on the corresponding scales.

Until now we have not specified the mass definition to be used in eq. (3). A natural choice for this mass is the pole mass $M$. In the numerical analysis we will first use the pole mass scheme to extract the pole masses. However, as the pole masses suffer from renormalon ambiguities [18, they can only be determined up to corrections of order $O\left(\Lambda_{Q C D}\right)$. Therefore, in the second part of our analysis we shall use the potential-subtracted (PS-) mass $m_{P S}$ [19]. From this mass definition we can obtain the $\overline{\mathrm{MS}}$-masses more accurately than from the pole mass scheme.

In the next section we will discuss the quantum mechanical sum rules. In section 3 we shall present the contributions from the threshold expansion in the framework of NRQCD. Here we will also define the potential-subtracted 
mass. The perturbative expansion will be derived in the following section. In section 5 and 6 we will discuss the nonperturbative contributions and the phenomenological spectral function. Then we shall explain the reconstruction of the spectral density. In the numerical analysis we will obtain the pole- and $\overline{\mathrm{MS}}$-masses from analyses in the pole- and PS-mass scheme respectively. The origin of different contributions to the error will be carefully investigated. After a comparison to other mass determinations we shall conclude with a summary and an outlook.

\section{Quantum mechanical sum rules for the Cou- lomb potential ${ }^{1}$}

Before studying the full quantum field theory case in detail, it will be instructive to first investigate the corresponding quantum mechanical system. Since it is possible to describe the system analytically, one can obtain a clearer picture of the structure of the method and the behaviour of the different contributions. Let us consider a system of two particles separated by a distance $\mathbf{x}$. The Schrödinger equation for stationary states takes the form

$$
\hat{H} \psi(\mathbf{x})=\left[-\frac{\Delta}{2 \mu}+V(\mathbf{x})\right] \psi(\mathbf{x})=E \psi(\mathbf{x}),
$$

where $\mu$ represents the reduced mass of the system. The Green's function is constructed with help of the resolvent operator $\hat{G}(E)=(\hat{H}-E)^{-1}$.

By introducing a full set of intermediate states we obtain the phenomenological side of the Green's function in position space:

$$
G(\mathbf{x}, \mathbf{y} ; E)=\langle\mathbf{x}|\hat{G}(E)| \mathbf{y}\rangle=\sum_{\alpha} \frac{\psi_{\alpha}(\mathbf{x}) \psi_{\alpha}^{*}(\mathbf{y})}{E_{\alpha}-E-i \epsilon}+\int d E^{\prime} \frac{\rho\left(\mathbf{x}, \mathbf{y} ; E^{\prime}\right)}{E^{\prime}-E-i \epsilon} .
$$

The sum runs over the discrete part of the spectrum, $\psi_{\alpha}$ being the eigenfunction to the eigenvalue $E_{\alpha}$. The integral is taken over the continuum part with the spectral density $\rho(\mathbf{x}, \mathbf{y} ; E)$. By taking the derivative at $\mathbf{x}=\mathbf{y}=0$, one can define a physical correlation function

$$
\begin{aligned}
M(E) & =\left[\frac{d}{d E} G(\mathbf{x}, \mathbf{y} ; E)\right]_{\mathbf{x}=\mathbf{y}=0} \\
& =\sum_{\alpha} \frac{\left|\psi_{\alpha}(0)\right|^{2}}{\left(E_{\alpha}-E-i \epsilon\right)^{2}}+\int d E^{\prime} \frac{\rho\left(E^{\prime}\right)}{\left(E^{\prime}-E-i \epsilon\right)^{2}} .
\end{aligned}
$$

\footnotetext{
${ }^{1}$ The author would like to thank Matthias Jamin who has initiated the investigations on the quantum mechanical sum rules and has contributed a substantial part to the development of these sum rules.
} 
Via a dispersion relation, the spectral density is related to the imaginary part of the Green's function $\rho(E)=\operatorname{Im} G(0,0 ; E) / \pi$. The second line of (7), constituting the phenomenological part, can be compared to a perturbative expansion of $M(E)$ thus representing the fundamental equation for the quantum mechanical sum rules [16, 20, 21]. So far the discussion has been general. Now we turn our attention to the Coulomb potential:

$$
V(r)=-\frac{\alpha}{r}, \quad E_{n}=-\frac{\mu \alpha^{2}}{2 n^{2}}, \quad\left|\psi_{n l m}(0)\right|^{2}=\delta_{l 0} \delta_{m 0} \frac{\mu^{3} \alpha^{3}}{\pi n^{3}} .
$$

In order to improve the predictive power it is convenient to formulate the sum rules in the framework of Borel or moment sum rules. Both cases will give valuable information: in the Borel sum rules we can relate $M(E)$ to the retarded propagator and in this way obtain explicit analytic expressions for the perturbative expansion. In the moment sum rules we can derive an equation for the mass and investigate the behaviour of the pole and continuum contributions for different values of $n$ and $\xi$.

\subsection{Borel sum rules}

In the Borel sum rules one applies a Borel transformation $\mathcal{B}_{\tau}$ to the correlation function. It is defined by

$$
\mathcal{B}_{\tau}=\lim _{-E, N \rightarrow \infty} \frac{(-E)^{N}}{\Gamma(N)} \frac{\partial^{N}}{(\partial E)^{N}}, \quad \frac{1}{\tau}=\frac{-E}{N} \text { fixed } .
$$

This enhances the importance of the ground state and improves the perturbative expansion. For the sum rule we will need the application of $\mathcal{B}_{\tau}$ on functions of the form $(x-E)^{-\alpha}$ :

$$
\mathcal{B}_{\tau} \frac{1}{(x-E)^{\alpha}}=\frac{\tau^{\alpha}}{\Gamma(\alpha)} e^{-x \tau}
$$

Now we define the Borel-transformed correlator as

$$
\begin{aligned}
M(\tau) & =\frac{1}{\tau^{2}} \mathcal{B}_{\tau} M(E)=\left\langle 0\left|e^{-\hat{H} \tau}\right| 0\right\rangle \\
& =\sum_{\alpha}\left|\psi_{\alpha}(0)\right|^{2} e^{-E_{\alpha} \tau}+\int_{0}^{\infty} d E^{\prime} \rho\left(E^{\prime}\right) e^{-E^{\prime} \tau} .
\end{aligned}
$$

Since the higher states are exponentially suppressed, the dominance of the ground state contribution for large $\tau$ is clearly improved. Let us now recall the definition of the retarded propagator:

$$
K\left(\mathbf{x}_{\mathbf{f}}, t_{f} ; \mathbf{x}_{\mathbf{i}}, t_{i}\right)=\left\langle\mathbf{x}_{\mathbf{f}}\left|e^{-i \hat{H}\left(t_{f}-t_{i}\right)}\right| \mathbf{x}_{\mathbf{i}}\right\rangle
$$


with $t_{f}>t_{i}$ and by analytic continuation one concludes immediately:

$$
M(\tau)=K(0,-i \tau ; 0,0)
$$

The known solution can be written as a product of the lowest order perturbation theory $M_{0}(\tau)$ and a function $F(\gamma)$ of the dimensionless variable $\gamma(\tau)[22]$

$$
\begin{aligned}
M(\tau) & =M_{0}(\tau) F(\gamma), \quad \gamma(\tau)=\alpha \sqrt{\frac{\mu \tau}{2}}, \quad \text { with } \\
M_{0}(\tau) & =\left(\frac{\mu}{2 \pi \tau}\right)^{3 / 2}, \\
F(\gamma) & =1+2 \sqrt{\pi} \gamma+\frac{2 \pi^{2}}{3} \gamma^{2}+4 \sqrt{\pi} \gamma^{3} \sum_{n=1}^{\infty} \frac{1}{n^{3}} e^{\gamma^{2} / n^{2}}(1+\operatorname{erf}(\gamma / n))
\end{aligned}
$$

with the function $\operatorname{erf}(x)=(2 / \sqrt{\pi}) \int_{0}^{x} \exp \left(-t^{2}\right) d t$. From this formula one can directly deduce the perturbative expansion as a power series in small $\gamma(\tau)$ :

$$
\begin{aligned}
M^{\text {Pert }}(\tau)= & M_{0}(\tau)\left(1+2 \sqrt{\pi} \gamma+\frac{2 \pi^{2}}{3} \gamma^{2}+4 \sqrt{\pi} \gamma^{3} \zeta(3)\right. \\
& \left.+8 \gamma^{4} \zeta(4)+4 \sqrt{\pi} \gamma^{5} \zeta(5)+O\left(\gamma^{6}\right)\right)
\end{aligned}
$$

On the other hand, $M(\tau)$ represents the sum of the poles and the continuum (11). By inserting the energy levels and wave functions from eq. (8) we can write the pole contributions as

$$
\begin{aligned}
M^{\text {Pole }}(\tau) & =M_{0}(\tau) 8 \sqrt{\pi} \gamma^{3} \sum_{n=1}^{\infty} \frac{1}{n^{3}} e^{\gamma^{2} / n^{2}} \\
& =M_{0}(\tau)\left(8 \sqrt{\pi} \gamma^{3} \zeta(3)+8 \sqrt{\pi} \gamma^{5} \zeta(5)+O\left(\gamma^{7}\right)\right)
\end{aligned}
$$

where the second line shows the expansion for small $\gamma$. The continuum contribution can be obtained from the difference of eqs. (15) and (16). Let us now assume a perturbative calculation order by order in the coupling constant. One would successively obtain the different orders in $\gamma^{n}$. Up to NNLO only the continuum contribution shows up. Then, at order $\gamma^{3}$, one gets a contribution from the poles $\sim 8 \sqrt{\pi} \gamma^{3} \zeta(3)$ which is partly cancelled by the continuum contribution $\sim-4 \sqrt{\pi} \gamma^{3} \zeta(3)$; likewise for higher orders. The poles, starting from the order $\gamma^{3}$, can thus be expected to be suppressed in the numerical evaluation. However, this conclusion is premature. As can be seen from eq. (16), the expression for $M^{\text {Pole }}$ contains an exponential in $\gamma$. 
Whereas for very small $\gamma$ the poles can be safely neglected, for large $\gamma$ these contributions grow exponentially and can far exceed the continuum part.

Let us finally note that the perturbative expansion is not only an expansion in $\alpha$, but in $\gamma=\alpha \sqrt{\mu \tau / 2}$ and thus depends on the Borel parameter $\tau$. The perturbative expansion and the relative size of the poles and the continuum will therefore depend on the sum rule window for $\tau$ chosen in the analysis. This behaviour is better discussed in the context of moment sum rules which closely resemble the field theory case at hand.

\subsection{Moment sum rules}

In the quantum mechanical Coulomb problem we define the moments as

$$
M_{n}(\xi)=\left.\frac{\pi}{n !} \frac{2}{\mu^{2} \alpha}\left(\mu \alpha^{2} \frac{d}{d E}\right)^{n} G(E)\right|_{E=\xi E_{1}}, \quad \xi>1 .
$$

Again, we allow for an arbitrary evaluation point $\xi$. A natural scale is given by the lowest bound state energy $E_{1}=-\mu \alpha^{2} / 2$. The parameter $\xi$ has been defined somewhat different as compared to eq. (31). The derivatives must be taken in an energy region below the poles where the Green's function is purely real. Here, we must therefore choose $\xi>1$ whereas in eq. (3) $\xi=0$ already represents a perturbative region. Solving the relevant Schrödinger equation, the radial Green's function is found to be [22]

$$
G(r, 0 ; E)=\frac{\mu k}{\pi} e^{-k r} \Gamma(1-\lambda) U(1-\lambda, 2 ; 2 k r)
$$

with the variables

$$
\lambda=\frac{\mu \alpha}{k} \quad \text { and } \quad k=\sqrt{-2 \mu(E+i \epsilon)} .
$$

$U(\alpha, \beta ; z)$ is the confluent hypergeometric function. $G(r, 0 ; E)$ is singular in the limit $r \rightarrow 0$, but the moments are finite and the first moment $M_{1}(\xi)$ is found to be

$$
M_{1}(\xi)=\left.\lambda\left(1+2 \lambda+2 \lambda^{2} \psi^{\prime}(1-\lambda)\right)\right|_{\lambda=1 / \sqrt{\xi}}
$$

The evaluation point $E=\xi E_{1}$ translates into $\lambda=1 / \sqrt{\xi}$. In this theoretical expression, all powers of $\lambda$ are resummed. Like the parameter $\gamma(\tau)$ in the Borel sum rules, here the true parameter for the perturbative expansion is $\lambda=\alpha / \sqrt{-2 E / \mu}$. The higher moments can be derived from a recursion relation:

$$
M_{n}(\xi)=\left.\frac{\lambda^{3}}{n} \frac{d}{d \lambda} M_{n-1}(\lambda)\right|_{\lambda=1 / \sqrt{\xi}}
$$


To derive the phenomenological parametrisation of the sum rules we need the spectral density for positive energies which can be obtained from

$$
\rho(E)=\frac{1}{\pi} \operatorname{Im} G(0,0 ; E) .
$$

$\operatorname{Im} G(r, 0 ; E)$ is finite in the limit $r \rightarrow 0$ since it is a physical quantity and gives the Sommerfeld factor

$$
\rho(E)=\frac{\alpha \mu^{2}}{\pi\left(1-e^{-\pi \alpha \sqrt{2 \mu / E}}\right)}, \quad E \geq 0 .
$$

Putting everything together, we obtain the phenomenological parametrisation

$$
\begin{aligned}
M_{n}^{\text {Phen }}(\xi)= & M_{n}^{\text {Poles }}(\xi)+M_{n}^{\text {Cont }}(\xi)=\sum_{k=1}^{\infty} \frac{2}{k^{3}\left(\frac{E_{k}}{\mu \alpha^{2}}+\frac{\xi}{2}\right)^{n+1}} \\
& +\int_{0}^{\infty} d x \frac{2}{\left(1-e^{-\pi \sqrt{2 / x}}\right)\left(x+\frac{\xi}{2}\right)^{n+1}} .
\end{aligned}
$$

Equating this quantity to the theoretical side (20121) establishes the sum rules. Using the exact formulas, this of course represents nothing but an identity. The method comes into play when only limited information on either part is available. It can then, for instance, be used to extract the lowest bound state energy by solving for $E_{1}$ as the higher bound states are strongly suppressed by the factor $1 / k^{3}$ in eq. (24). Likewise, assuming that the energy levels are known, one can solve for the mass $\mu$ since the dominant dependence on the mass originates from $E_{1}$ :

$$
\mu=\frac{E_{1}}{\alpha^{2}}\left[\left(\frac{2}{M_{n}^{\text {Theo }}-M_{n}^{\text {Cont }}-M_{n}^{\text {HigherPoles }}}\right)^{\frac{1}{n+1}}-\frac{\xi}{2}\right]^{-1}
$$

Since the higher poles $M_{n}^{\text {HigherPoles }}$ contain $E_{k}$ and $\mu$ as parameters, eq. (25) represents a fast converging self-consistency equation for $\mu$. Here we shall not discuss the applications of this sum rule, but finally turn our attention to the behaviour of the pole and continuum contributions.

To this end, we again take a closer look to eq. (24). Both contributions depend in a similar way on $n$ and $\xi$. But whereas the integration runs over positive values of $x \geq 0$, the energy levels $E_{k}$ are located below threshold. Let us investigate the behaviour of the moments on $n$ for fixed $\xi$. For low values of $n$ the higher poles and high energy part of the continuum integration can 


\begin{tabular}{|c||c|c|c|c||c|c|c|c|}
\hline \multicolumn{1}{|c||}{} & \multicolumn{5}{c||}{$\xi=4$} & \multicolumn{5}{c|}{$\xi=10$} \\
\hline$n$ & 1 & 3 & 5 & 7 & 1 & 3 & 5 & 7 \\
\hline$M_{n}^{\text {Poles }} / M_{n}^{\text {Theo }}$ & 0.45 & 0.83 & 0.94 & 0.97 & 0.16 & 0.49 & 0.67 & 0.78 \\
$M_{n}^{\text {Cont }} / M_{n}^{\text {Theo }}$ & 0.55 & 0.17 & 0.06 & 0.03 & 0.84 & 0.51 & 0.33 & 0.22 \\
\hline \hline \multicolumn{1}{|c||}{} & \multicolumn{4}{c||}{$n=3$} & \multicolumn{5}{c|}{$n=7$} \\
\hline$\xi$ & 2 & 4 & 10 & 50 & 2 & 4 & 10 & 50 \\
\hline$M_{n}^{\text {Poles }} / M_{n}^{\text {Theo }}$ & 0.98 & 0.83 & 0.49 & 0.10 & 0.9998 & 0.97 & 0.78 & 0.26 \\
$M_{n}^{\text {Cont }} / M_{n}^{\text {Theo }}$ & 0.02 & 0.17 & 0.51 & 0.90 & 0.0002 & 0.03 & 0.22 & 0.74 \\
\hline
\end{tabular}

Table 1: Relative size of pole and continuum contributions to the theoretical moments $M_{n}^{\text {Theo }}=M_{n}^{\text {Poles }}+M_{n}^{\text {Cont }}$ for different values of $n$ at fixed $\xi$ and for different $\xi$ at fixed $n$.

have a significant influence. When we proceed to larger $n$ we enhance the threshold region in the continuum integration and the lowest pole in the sum. Taking now $n$ fixed we see that the variation of $\xi$ can drastically change the relative size of both parts: Since the moments have a singularity at $\xi=1$ from $E_{1}$, values of $\xi$ only slightly larger than one will lead to a complete dominance of the first bound state on the sum rules. Larger $\xi$ enhance the higher bound states and also the continuum part gets more and more important. The results are summarised in table 1. We have depicted the pole and continuum contributions for different values of $n$ and $\xi$. One should keep in mind that the relative size of the pole and continuum contributions does not directly depend on the physical system under investigation but rather on the values of $n$ and $\xi$ chosen for the analysis; namely the poles can depend strongly on these parameters. However, it is important to note that the main mass dependence originates from the first bound states since the continuum is largely independent of the mass. This remains true even in a region where the continuum dominates the moments. Therefore, to obtain good accuracy when extracting e.g. the ground state energy or the mass it is advantageous to use low $\xi$ and high $n$. Then the contribution from $E_{1}$ will dominate the sum rules. Unfortunately, in this region also the perturbative expansion converges more slowly. The perturbative series behaves better for lower $n$ and also for higher $\xi$ since this parameter enters directly in the expansion variable $\lambda=1 / \sqrt{\xi}$. Therefore, in practical applications where the exact solutions are not known, one must carefully choose a range of values for $n$ and $\xi$ such that the theoretical calculation can be reliably trusted without loosing sensitivity on the parameters one would like to extract. These considerations will be made more explicit in the numerical analysis. Now we will discuss 
the Coulomb contributions in the full field theory system.

\section{Coulomb resummation}

The theory of NRQCD provides a consistent framework to treat the problem of heavy quark-antiquark production close to threshold. The contributions can be described by a nonrelativistic Schrödinger equation and systematically calculated in time-independent perturbation theory (TIPT) 23]. At NNLO the factorised formulation has first been shown in 24. The correlator is expressed in terms of a Green's function $G(k)=G(0,0, k)$ :

$$
\Pi(s)=\frac{N_{c}}{2 M^{2}}\left(C_{h}\left(\alpha_{s}\right) G(k)+\frac{4 k^{2}}{3 M^{2}} G_{C}(k)\right),
$$

where $N_{c}$ is the number of colours, $k=\sqrt{M^{2}-s / 4}$ and $M$ represents the pole mass 13. First we will present the method in the pole mass scheme and afterwards discuss the PS-scheme. The constant $C_{h}\left(\alpha_{s}\right)$ is a perturbative coefficient needed for the matching between the full and the nonrelativistic theory. It naturally depends on the hard scale and is given by [24]

$$
\begin{aligned}
C_{h}\left(\alpha_{s}\right)= & 1-4 C_{F} \frac{\alpha_{s}\left(\mu_{\text {hard }}\right)}{\pi}+C_{h}^{(2)} C_{F}\left(\frac{\alpha_{s}\left(\mu_{\text {hard }}\right)}{\pi}\right)^{2}, \\
C_{h}^{(2)}= & \left(\frac{39}{4}-\zeta(3)+\frac{4 \pi^{2}}{3} \ln 2-\frac{35 \pi^{2}}{18}\right) C_{F}-\left(\frac{151}{36}+\frac{13}{2} \zeta(3)\right. \\
& \left.+\frac{8 \pi^{2}}{3} \ln 2-\frac{179 \pi^{2}}{72}\right) C_{A}+\left(\frac{44}{9}-\frac{4 \pi^{2}}{9}+\frac{11}{9} n_{f}\right) T \\
& +2 b_{0} \ln \left(\frac{M}{\mu_{\text {hard }}}\right)+\pi^{2}\left(\frac{2}{3} C_{F}+C_{A}\right) \ln \left(\frac{M}{\mu_{\text {fac }}}\right),
\end{aligned}
$$

where $C_{F}=4 / 3, C_{A}=3, T=1 / 2$ and $b_{0}=11-2 n_{f} / 3 . G_{C}(k)$ represents the Coulomb Green's function and reads

$$
G_{C}(k)=-\frac{C_{F} \alpha_{s} M^{2}}{4 \pi}\left[\frac{k}{C_{F} \alpha_{s} M}+\ln \left(\frac{k}{\mu_{f a c}}\right)+\gamma_{E}+\Psi\left(1-\frac{C_{F} \alpha_{s} M}{2 k}\right)\right] .
$$

The contributions from NRQCD are summarised in the potential. The Green's function obeys the corresponding Schrödinger equation

$$
\left(-\frac{\Delta_{x}}{M}+V_{C}(x)+\Delta V(x)+\frac{k^{2}}{M}\right) G(\mathbf{x}, \mathbf{y}, k)=\delta^{(3)}(\mathbf{x}-\mathbf{y}) .
$$


Here $V_{C}(x)=-C_{F} \alpha_{s} /|\mathbf{x}|$ represents the Coulomb potential and $\Delta V(x)$ contains the NLO and NNLO corrections. The explicit form of the potential is given in the appendix. The full Green's function can be derived from TIPT and to first order one obtains

$$
\begin{aligned}
G(0,0, k) & =G_{C}(0,0, k)+\Delta G(0,0, k), \\
\Delta G(0,0, k) & =-\int d^{3} \mathbf{x} G_{C}(0, \mathbf{x}, k) \Delta V(x) G_{C}(\mathbf{x}, 0, k) .
\end{aligned}
$$

To be consistent to NNLO one also has to apply second order perturbation theory to the one-loop potential. Details about this procedure can be found in [13, 14]. To calculate the moments from the Green's function we will directly perform the derivatives at $s=-4 M^{2} \xi$ according to eq. (3). Since the Green's function is known analytically [13] as a function of $k=k(s)$, this can be done numerically. In this way we take advantage of the fact that the perturbative expansion parameter depends on the evaluation point. The expansion of the moments shows the same behaviour as has already been discussed in the quantum mechanical sum rules (2021). There the expansion parameter of the moments is $\lambda=1 / \sqrt{\xi}$ and so higher values for $\xi$ improve the perturbative series. The resulting moments include both the pole and the continuum contributions.

The moments depend on three scales: The hard scale $\mu_{\text {hard }} \sim M$ enters in the coefficient $C_{h}$ of eq. (26). This scale is also needed for the perturbative expansion which will be discussed in the next section. The soft scale $\mu_{\text {soft }} \sim$ $M v$ is a typical scale for nonrelativistic processes and the relevant scale for the expansion of the Green's function. Furthermore, the factorisation scale $\mu_{\text {fac }}$ separates the contributions of large and small momenta and plays the role of an infrared cutoff. As we perform the calculation only up to NNLO we are left with a residual dependence on these three scales. In fact, the dependence of the mass on the scales, especially on $\mu_{\text {soft }}$, is rather large and will give the dominant source of the error. To obtain the central values for the masses we will use a set of values for $\mu_{\text {soft }}, \mu_{\text {fac }}$ and $\mu_{\text {hard }}$ according to the physical expectations from the charmonium and bottomium systems. The error will then be estimated by allowing for sufficiently large variations of these scales.

Though the full theoretical moments from resummation can thus be determined, we are also interested in the pole and continuum contributions separately. First, we want to analyse them independently and estimate their contribution to the error. Second, in our numerical analysis we will reconstruct the spectral density above threshold. At low velocities it is given by the imaginary part of the nonrelativistic Green's function.

In principle, the expressions for the energies and decay widths of the poles 
have been calculated at NNLO. One could then deduce their contribution to the moments as in eq. (24). But in this method the contributions have to be calculated near threshold and thus show large corrections already for the bottomium and cannot be trusted for the charmonium.

Therefore we will choose a different method of evaluation [17, 25. By using a dispersion relation, we derive the continuum from the imaginary part of the correlator. From the difference we can then obtain the pole contributions:

$$
\begin{aligned}
\mathcal{M}_{n}^{\text {Poles }}= & \left.\frac{12 \pi^{2}}{n !}\left(4 M^{2} \frac{d}{d s}\right)^{n} \Pi(s)\right|_{s=-4 M^{2} \xi} \\
& -12 \pi\left(4 M^{2}\right)^{n} \int_{4 M^{2}}^{\infty} d s \frac{\operatorname{Im} \Pi(s)}{\left(s+4 M^{2} \xi\right)^{n+1}} .
\end{aligned}
$$

Nevertheless, for values of $n$ and $\xi$ used in our analysis, the poles will give the largest contribution to the theoretical moments and thus the dependence on the scales will remain relatively strong. In the numerical analysis we will give a detailed account on the size and behaviour of these contributions.

Now we investigate the spectral density from the continuum part. We discuss the charmonium system since the differences in the expansion can be seen more clearly than in the bottomium which shows a faster convergence. In figure 1 we have displayed the spectral density times the weight factor for the different orders. The area under the spectral density is directly proportional to the moments. We have chosen moments and scales typical for the numerical analysis, $n=5, \xi=0.5, \mu_{\text {soft }}=1.1 \mathrm{GeV}, \mu_{f a c}=1.45 \mathrm{GeV}$ and $\mu_{\text {hard }}=1.75 \mathrm{GeV}$. The dotted line represents the LO, the dashed line includes the NLO and the solid line represents the full NNLO result. One can see that the expansion of the moments converges well in the low velocity region. But when higher $v$ are used, resummation is not capable to incorporate the correct high energy behaviour. Therefore, when we reconstruct the complete spectral density, we will use the resummed spectral density only below a separation velocity $v<v_{\text {sep }}$ where the expansion can be trusted.

Part of the large corrections to the potential is not inherent to the bound state system but to the definition of the pole mass. In [26, 19] it was observed that the long distance sensitivity in the coordinate space potential cancels to all orders in perturbation theory with the long distance sensitivity in the pole mass. Therefore a new mass definition has been proposed, the potentialsubtracted mass $m_{P S}$, where the potential below a separation scale $\mu_{\text {sep }}$ is subtracted:

$$
m_{P S}\left(\mu_{\text {sep }}\right)=M-\delta m\left(\mu_{\text {sep }}\right), \quad \delta m\left(\mu_{\text {sep }}\right)=-\frac{1}{2} \int_{|\mathbf{q}|<\mu_{\text {sep }}} \frac{d^{3} q}{(2 \pi)^{3}} V(q) .
$$




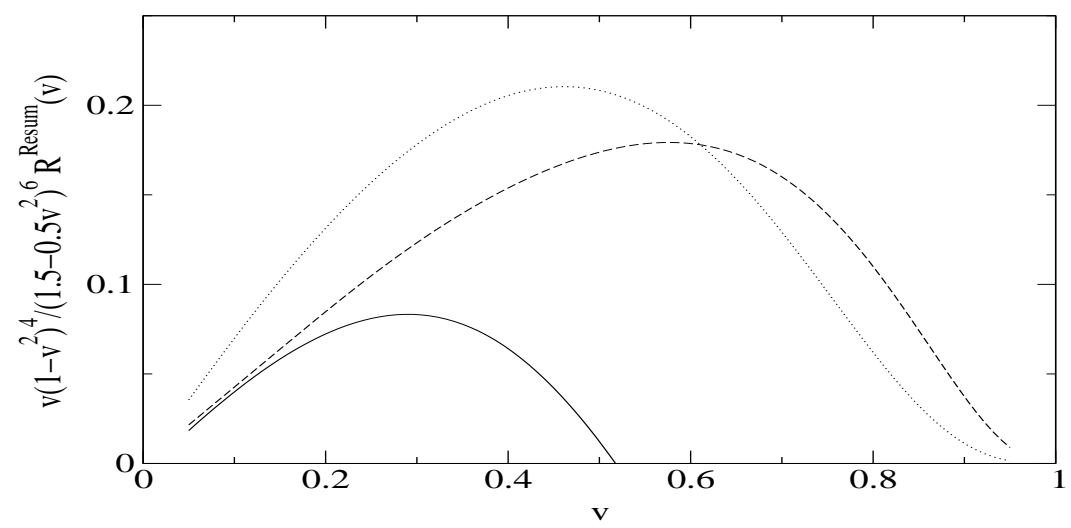

Figure 1: Resummed spectral density times the weight factor corresponding to $n=5$ and $\xi=0.5$ in LO (dotted), NLO (dashed) and NNLO (solid) at typical scales for the charmonium $\mu_{\text {soft }}=1.1 \mathrm{GeV}, \mu_{\text {fac }}=1.45 \mathrm{GeV}$ and $\mu_{\text {hard }}=1.75 \mathrm{GeV}$.

The subtracted potential $V\left(r, \mu_{\text {sep }}\right)$ is then defined by

$$
V\left(r, \mu_{\text {sep }}\right)=V(r)+2 \delta m\left(\mu_{\text {sep }}\right) .
$$

When using these definitions the Schrödinger equation for the Green's function takes it usual form where the pole mass is substituted by the PS-mass and the potential by $V\left(r, \mu_{\text {sep }}\right)$ [19]. Since the renormalon contributions have been subtracted from the potential, the convergence of the expansion is improved and the strength of the potential is reduced. The developed methods for the evaluation of the Green's function can again be employed and the additional contribution is absorbed in a shift of the energy.

We then express all moments in terms of the PS-mass and perform the analysis for $m_{P S}$. The PS-mass is perturbatively related to the $\overline{\mathrm{MS}}$-mass:

$$
\begin{aligned}
m_{P S}\left(\mu_{\text {sep }}\right)= & m\left[1+\frac{\alpha_{s}(m)}{\pi}\left(k_{1}-C_{F} \frac{\mu_{s e p}}{m}\right)+\left(\frac{\alpha_{s}(m)}{\pi}\right)^{2}\left(k_{2}-C_{F} \frac{\mu_{\text {sep }}}{m}\right.\right. \\
& \left.\left.\times \frac{w_{1}\left(m, \mu_{\text {sep }}\right)}{4}\right)+\left(\frac{\alpha_{s}(m)}{\pi}\right)^{3}\left(k_{3}-C_{F} \frac{\mu_{s e p}}{m} \frac{w_{2}\left(m, \mu_{s e p}\right)}{16}\right)\right], \\
k_{1}= & C_{F}, \quad k_{2}=13.443-1.041 n_{f} \\
k_{3}= & 190.595-26.655 n_{f}+0.653 n_{f}^{2}
\end{aligned}
$$

where $m=m_{\overline{\mathrm{MS}}}\left(m_{\overline{\mathrm{MS}}}\right)$ is the $\overline{\mathrm{MS}}$-mass evaluated at its own scale. The 
functions $w_{1}$ and $w_{2}$ can be found in the appendix where also a more complete list of formulas to the PS-mass is given.

The definition of the PS-mass and its relation to the $\overline{\mathrm{MS}}$-mass depends on $\mu_{\text {sep }}$. This scale must be taken large enough to guarantee a perturbative relation between the masses. At the same time it should be chosen smaller than a typical nonrelativistic scale as not to affect the threshold behaviour:

$$
\Lambda_{Q C D}<\mu_{\text {sep }}<M \cdot v
$$

In the numerical analysis we will see that the use of the PS-mass improves the error of the $\overline{\mathrm{MS}}$-mass. To estimate the error on $\mu_{\text {sep }}$, we shall also vary this scale in appropriate ranges.

\section{Perturbative expansion}

The perturbative spectral function $R^{\text {Pert }}(s)$ can be expanded in powers of the strong coupling constant $a=\alpha_{s} / \pi$,

$$
R^{\text {Pert }}(s)=R^{(0)}(s)+a R^{(1)}(s)+a^{2} R^{(2)}(s)+O\left(a^{3}\right) .
$$

From this expression the corresponding moments $\mathcal{M}_{n}^{\text {Pert }}(\xi)$ can be calculated via the integral of eq. (44). For the first two terms, analytic expressions are available [27] and they read

$$
\begin{aligned}
R^{(0)}(v)= & \frac{3}{2} v\left(3-v^{2}\right) \\
R^{(1)}(v)= & 2\left(1+v^{2}\right)\left(3-v^{2}\right)\left[4 \operatorname{Li}_{2}(p)+2 \operatorname{Li}_{2}(-p)+\ln (p)(\ln (1+p)\right. \\
& +2 \ln (1-p))]-4 v\left(3-v^{2}\right)(\ln (1+p)+2 \ln (1-p)) \\
& -\frac{1}{4}(1-v)\left(33-39 v-17 v^{2}+7 v^{3}\right) \ln (p)+\frac{3}{2} v\left(5-3 v^{2}\right)
\end{aligned}
$$

where $p=(1-v) /(1+v)$ and $\operatorname{Li}_{2}(\mathrm{z})$ is the dilogarithmic function. The corresponding formulas for $\Pi^{(0)}$ and $\Pi^{(1)}$ can, for instance, be found in [28]29]. $R^{(2)}(s)$ is still not fully known analytically. We employ a method based on Padé-approximants to construct the spectral density in the full energy range [30, 31]. It uses available information around $q^{2}=0$, at threshold and in the high energy region. It has the advantage that it gives a good description until relatively close to threshold. In this region the moments show a strong variation for relatively small changes of the mass. A pure high energy expansion would only be valid for large values of the velocity and a matching between the threshold and the perturbative region would be less reliable. 


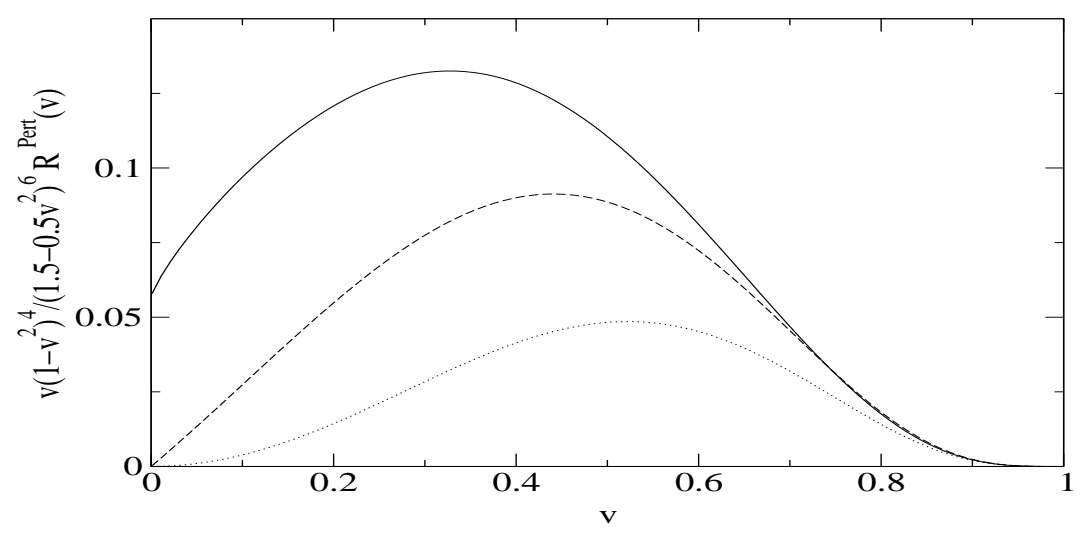

Figure 2: Perturbative spectral density times the weight factor corresponding to $n=5$ and $\xi=0.5$ in LO (dotted), NLO (dashed) and NNLO (solid) for $\mu_{\text {hard }}=1.75 \mathrm{GeV}$.

To illustrate the perturbative convergence we compare the different orders for the charmonium as in the last section for the resummed spectral density. In figure 2 we have displayed the spectral density times the weight factor, again for values of $n=5, \xi=0.5$ and $\mu_{\text {hard }}=1.75 \mathrm{GeV}$. The expansion converges well in the high velocity region. As we approach lower $v$, the expansion cannot be trusted since singular terms in $v$ appear which have to be resummed. These are included in the resummed spectral density which sums up terms of order $\alpha_{s}^{n} / v^{n-k}$, for $n \geq 0$ and $k=1,2,3$. The leading term in the perturbative spectral density at NNLO has a singular behaviour $\sim \alpha_{s}^{2} / v$, but its contribution to the moments remains finite since the weight function contains a factor of $v$. Consequently, the graph at NNLO in figure 2 starts with a constant set-off at $v=0$.

As will be explained in more detail in section 7 , for the perturbative moments we will therefore mainly use the spectral density above a separation velocity $v>v_{\text {sep }}$ with $v_{\text {sep }} \approx 0.4$. In table 2 we compare the behaviour of the moments for different values of $\xi$ and $v_{\text {sep }}$. The higher $v_{\text {sep }}$ and $\xi$ one chooses the more one approaches the perturbative region and the expansion improves. For typical values of the analysis, $v_{s e p}=0.4$ and $\xi=0.5$, the convergence is under good control.

To calculate the moments in the PS-scheme we can use the same integration formula (4) as in the pole mass scheme, but now the spectral density $R(s)$ is evaluated at the velocity $v=\sqrt{1-4 m_{P S}^{2} / s}$ and the start of the 


\begin{tabular}{|c|c|c|c|c|c|}
\hline \multicolumn{6}{|c|}{$\xi=0$} \\
\hline \multicolumn{2}{|c|}{$v_{s e p}$} & 0 & 0.2 & 0.4 & 0.6 \\
\hline \multirow{3}{*}{$\mathcal{M}_{5}^{\text {Pert }}$} & $\mathrm{LO}$ & 0.31 & 0.29 & 0.18 & 0.055 \\
\hline & NLO & 0.72 & 0.60 & 0.32 & 0.081 \\
\hline & NNLO & 1.29 & 0.87 & 0.38 & 0.085 \\
\hline \multicolumn{6}{|c|}{$\xi=0.5$} \\
\hline \multicolumn{2}{|c|}{$v_{s e p}$} & 0 & 0.2 & 0.4 & 0.6 \\
\hline \multirow{3}{*}{$\mathcal{M}_{5}^{\text {Pert }}$} & $\mathrm{LO}$ & 0.046 & 0.044 & 0.033 & 0.014 \\
\hline & NLO & 0.096 & 0.085 & 0.054 & 0.020 \\
\hline & NNLO & 0.15 & 0.12 & 0.064 & 0.021 \\
\hline \multicolumn{6}{|c|}{$\bar{\xi}=1$} \\
\hline \multicolumn{2}{|c|}{$v_{\text {sep }}$} & 0 & 0.2 & 0.4 & 0.6 \\
\hline \multirow{3}{*}{$\mathcal{M}_{5}^{\text {Pert }}$} & $\mathrm{LO}$ & 0.012 & 0.011 & 0.0092 & 0.0048 \\
\hline & NLO & 0.023 & 0.021 & 0.015 & 0.0068 \\
\hline & NNLO & 0.034 & 0.027 & 0.017 & 0.0070 \\
\hline \multicolumn{6}{|c|}{$\xi=2$} \\
\hline \multicolumn{2}{|c|}{$v_{s e p}$} & 0 & 0.2 & 0.4 & 0.6 \\
\hline \multirow{3}{*}{$\mathcal{M}_{5}^{\text {Pert }}$} & $\mathrm{LO}$ & 0.0017 & 0.0017 & 0.0015 & 0.00093 \\
\hline & NLO & 0.0030 & 0.0028 & 0.0023 & 0.0013 \\
\hline & NNLO & 0.0041 & 0.0035 & 0.0025 & 0.0013 \\
\hline
\end{tabular}

Table 2: Perturbative moments at LO, NLO and NNLO with $\mu_{\text {hard }}=1.75$ $\mathrm{GeV}$ and $n=5$ for different values of $\xi$. The moments are calculated only from the perturbative spectral density above $v>v_{\text {sep }}$. 

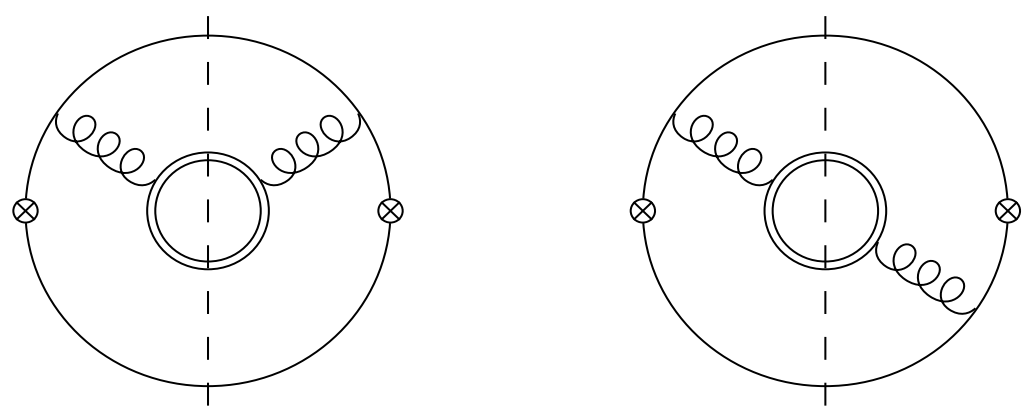

Figure 3: Diagrams at $O\left(\alpha_{s}^{2}\right)$ from the light quark correlator contributing to heavy quark production.

integration $v_{\text {sep }}$ must be transformed to this scheme as well.

At $O\left(\alpha_{s}^{2}\right)$ it is no longer true that heavy quark production originates exclusively from the heavy quark correlator. Also the light quark correlator includes a four fermion cut with a heavy quark pair radiated off the light quarks [32]. The corresponding diagrams are shown in fig. 3. When these contributions are included in the measurements, which of course depends on the experimental setup, they should be considered in the theoretical side as well. The diagrams have been calculated in ref. [33]. The resulting expressions can be split in such a way that they allow to introduce Coulomb resummation effects of the heavy quark pair in a straightforward way. However, as was discussed in [32, the heavy quark pair is produced in a colour octet state from the gluon splitting. In this case the potential becomes repulsive and the cross section decreases close to threshold. For high energies the diagram gives the same contribution as the diagram with the light and heavy quark lines interchanged. Since the main contribution of this diagram comes from the perturbative region, its $O\left(\alpha_{s}^{2}\right)$ contribution to the moments is suppressed and has a typical relative size of $2 \cdot 10^{-5}$. The shift in the final value for the $\overline{\mathrm{MS}}$-bottom quark mass then amounts to $\Delta m_{b}\left(m_{b}\right) \approx 9$ $\mathrm{keV}$ and can be safely neglected within the uncertainty of this analysis. To conclude the theoretical side of the correlator we now discuss the condensate contributions.

\section{Condensate contributions}

The nonperturbative effects on the vacuum correlator are parametrised by the condensates. The leading term is the gluon condensate contribution. It has been calculated up to next-to-leading order 34] and can be written as 
follows:

$$
\Pi_{F F}^{\text {Cond }}(s)=\frac{\langle a F F\rangle}{16 M^{4}}\left(C_{F F}^{(0)}(s)+a C_{F F}^{(1)}(s)+O\left(a^{2}\right)\right) .
$$

The analytic form of the functions $C_{F F}^{(0)}(s)$ and $C_{F F}^{(1)}(s)$ can be found in [34]. The results have been calculated in dimensional regularisation with the pole mass $M$. Using a different mass prescription like the PS-mass, $C_{F F}^{(1)}(s)$ has to change accordingly. In our analysis below, we employ a value of $\langle a F F\rangle=$ $0.024 \pm 0.012 \mathrm{GeV}^{4}$ for the gluon condensate.

Furthermore, in 35, 36, 37 the dimension 6 and 8 condensate contributions have been calculated. However, this has been done only for moments at $\xi=0$ and therefore only in this case we take them into account. For typical values of charmonium scales, $\mu_{\text {hard }}=M=1.75 \mathrm{GeV}$, their contribution is $5 \%-10 \%$ of the leading gluon condensate. In fact, as will be shown in the numerical analysis, the absolute contribution of the condensates to the full theoretical moments is small, both for the upsilon and the charmonium. Whereas former sum rule analyses for the charmonium have emphasised the significance of these nonperturbative contributions, their relative suppression in this work is due to three reasons: First, the absolute value of the theoretical moments increases from the Coulomb resummation. Then we evaluate the moments at larger $\xi$ and smaller $n$ where the nonperturbative contributions are relatively small. Finally, since we obtain a larger pole mass than former analyses, the condensates, starting with a power of $1 / M^{4}$, are suppressed further.

\section{Phenomenological spectral function}

Experimentally, the six lowest lying $\psi-$ and $\Upsilon$-resonances have been observed. To obtain the phenomenological content of the spectral function we use the narrow-width approximation for the resonances

$$
R_{k}(s)=\frac{9 \pi}{\bar{\alpha}^{2} Q_{c, b}^{2}} \Gamma_{k}\left(\psi_{k}, \Upsilon_{k} \rightarrow e^{+} e^{-}\right) E_{k} \delta\left(s-E_{k}^{2}\right)
$$

where $Q_{c, b}$ represents the electric charge of the charm or bottom quark. $\bar{\alpha}$ denotes the running QED coupling evaluated at a scale around the resonance mass. For the charm this corresponds to the fine structure constant $\alpha=1 / 137.04$ whereas for the bottom widths the Review of Particle Properties [1] has used $\bar{\alpha}^{2}=1.07 \alpha^{2}$ and we will do so accordingly. The narrow-width approximation provides an excellent description of these states since the full hadronic widths are much smaller than the masses. The values for the masses 


\begin{tabular}{|c||c|c|c|}
\hline$k$ & 1 & 2 & 3 \\
\hline \hline$E_{k}[\mathrm{GeV}]$ & 3.097 & 3.686 & 3.770 \\
\hline$\Gamma_{k}[\mathrm{keV}]$ & $5.26 \pm 0.37$ & $2.12 \pm 0.18$ & $0.24 \pm 0.05$ \\
\hline \hline$k$ & 4 & 5 & 6 \\
\hline \hline$E_{k}[\mathrm{GeV}]$ & 4.040 & 4.159 & 4.415 \\
\hline$\Gamma_{k}[\mathrm{keV}]$ & $0.75 \pm 0.15$ & $0.77 \pm 0.23$ & $0.47 \pm 0.10$ \\
\hline
\end{tabular}

Table 3: Masses and electronic widths of the first six $\psi_{k}$-resonances.

\begin{tabular}{|c||c|c|c|}
\hline$k$ & 1 & 2 & 3 \\
\hline \hline$E_{k}[\mathrm{GeV}]$ & 9.460 & 10.023 & 10.355 \\
\hline$\Gamma_{k}[\mathrm{keV}]$ & $1.32 \pm 0.07$ & $0.52 \pm 0.04$ & $0.48 \pm 0.08$ \\
\hline \hline$k$ & 4 & 5 & 6 \\
\hline \hline$E_{k}[\mathrm{GeV}]$ & 10.580 & 10.865 & 11.019 \\
\hline$\Gamma_{k}[\mathrm{keV}]$ & $0.25 \pm 0.03$ & $0.31 \pm 0.07$ & $0.13 \pm 0.03$ \\
\hline
\end{tabular}

Table 4: Masses and electronic widths of the first six $\Upsilon_{k}$-resonances.

and electronic widths are collected in tables 3 and 4 For our numerical analysis the errors on the masses can be safely neglected and have thus not been listed. It should be kept in mind that the moments from the experimental resonances are not identical to the ones obtained from the poles of the Green's function in section 3 which represent a summation of a special kind of theoretical contributions.

For the upsilon system, the hadronic continuum is not measured with sufficient accuracy so we use the assumption of quark-hadron duality and integrate the theoretical spectral density above a continuum threshold $s_{0}$ :

$$
\frac{\mathcal{M}_{b, n}}{\left(4 M_{b}^{2}\right)^{n}}=\frac{9 \pi}{\bar{\alpha}^{2} Q_{b}^{2}} \sum_{k=1}^{6} \frac{\Gamma_{b, k} E_{b, k}}{\left(E_{b, k}^{2}+4 M_{b}^{2} \xi\right)^{n+1}}+\int_{s_{0}}^{\infty} d s \frac{R_{b}^{R c s t r}(s)}{\left(s+4 M_{b}^{2} \xi\right)^{n+1}} .
$$

For the parametrisation of the spectral density we use the reconstructed spectral density $R_{b}^{R c s t r}(s)$ which will be discussed in the next section. The continuum from open $B$ production sets in at $\sqrt{s}=2 M_{B}=10.56 \mathrm{GeV}$ just below the 4th resonance. In the upsilon system the resonances are relatively dominant. The start of the continuum threshold $s_{0}$ should thus in principle be given by the mass of the 7th resonance. Nevertheless, when we take into account only the first 3 resonances and a continuum threshold $s_{0}$ typically $250 \mathrm{MeV}$ above the 3th resonance and compare the result to an evaluation 


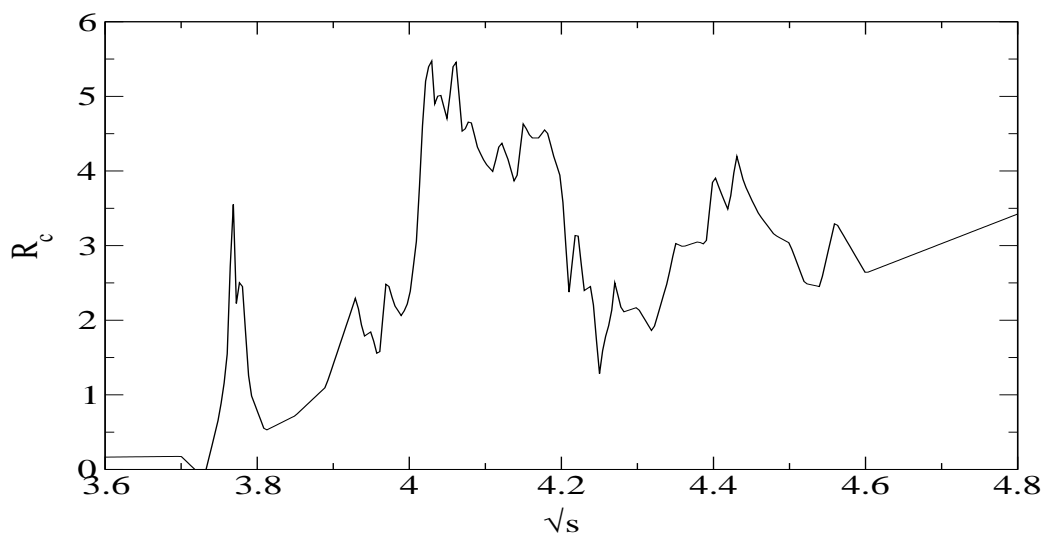

Figure 4: $R_{c}(s)$ from 38 .

with all 6 resonances and $s_{0}$ above the 6 th resonance we miss in the latter a contribution of $30 \%$ from the continuum. It seems natural to assume that this contribution originates from open $B$ production. To account for this contribution we lower the value of $s_{0}$ to $\sqrt{s_{0}}=11.0 \mathrm{GeV}$. To estimate the error we vary $s_{0}$ between $10.8 \mathrm{GeV}<\sqrt{s_{0}}<11.2 \mathrm{GeV}$. To be conservative, in the analysis we also check the influence on the error if we remove the resonances above the continuum.

Above the charmonium threshold recent measurements have improved the phenomenological situation significantly 38. 85 data points have been taken in the region between $2.0 \mathrm{GeV}<\sqrt{s_{0}}<4.8 \mathrm{GeV}$ with an average precision of $6.6 \%$. The continuum threshold starts at $\sqrt{s}=2 M_{D}=3.73 \mathrm{GeV}$. From the measured spectral density the light quark contributions must be subtracted. At this energy the light quarks can be safely assumed to be massless and the high energy approximation [39] provides a good description. The resulting spectral density is shown in figure 4. At energies above the data points we again use the reconstructed spectral density. Since the resonances 3-6 are well reproduced by the data, we only add the first two resonances below the continuum threshold. The error from the data turns out to be small compared to the theoretical uncertainties.

It is interesting to compare the measured cross section to the predictions from quark-hadron-duality. In average, the reconstructed spectral density lies above the data points. This should be no surprise as the OPE demands an equality of the theoretical and phenomenological moments only for the full correlator which also includes the pole contributions. Since the lowest poles 
are very dominant on the phenomenological side they are compensated by a larger theoretical spectral density for intermediate values of $s$. Consequently, one should take care when describing the phenomenological spectral density by the perturbative one, in particular, the choice of the integration point $s_{0}$ could depend on the values of $\xi$ and $n$. A more detailed description of the charmonium cross section and the accuracy of quark-hadron-duality is presented in [40]. Now we explain how to construct a theoretical spectral density for the full energy range.

\section{Reconstruction of the spectral density}

Besides the contributions from the poles of the Green's function and the condensates, the theoretical part of the correlator contains the spectral density above threshold. Now we discuss the different parts of the spectral density.

For high velocities the spectral density is well described by the perturbative expansion. As explained in section 4 we have used a method which allows a good approximation until relatively close to threshold. The resummed spectral density, on the other hand, gives a good description for low values of $v$, but it fails to describe the high energy part. For these reasons, we introduce a separation velocity $v_{s e p}$. Above $v_{s e p}$ we only use the perturbative spectral density. Below $v_{\text {sep }}$ we essentially take the resummed spectral density. The perturbative expansion has singular terms in $v$ which are included in the resummed spectral density, but it also contains contributions from higher powers in $v$ which can be isolated by subtracting the double counted terms and these contributions will be added to the resummed spectral density below $v_{\text {sep }}$. In fig. 5 we have displayed the charmonium spectral density from the different contributions as a function of $v$. This representation expands the threshold region. The dotted line represents the perturbative expansion at NNLO. The dashed line is the resummed spectral density and the dashed-dotted line the reconstructed spectral density. For the charmonium system, there exists a range of intermediate values of $v$ where neither the perturbative expansion nor the resummation can be trusted. Indeed, it can be clearly seen that the reconstructed spectral density shows a gap at the separation velocity. Since this gap is not physical but a result of the mismatch between the two energy regions we can try to construct a more physical spectral density which interpolates smoothly between small and large $v$. We can construct this interpolating spectral density from $R^{\text {Resum }}$ and $R^{\text {Pert }}$ between the two velocities $v_{1}=0.2$ and $v_{2}=0.6$ with $R^{\text {Inter }}=R^{\text {Resum }}\left(v_{2}^{2}-v^{2}\right) /\left(v_{2}^{2}-v_{1}^{2}\right)+R^{\text {Pert }}\left(v^{2}-v_{1}^{2}\right) /\left(v_{2}^{2}-v_{1}^{2}\right)$. There is no explicit argument for a specific choice of $R^{\text {Inter }}$ except that it should give a 


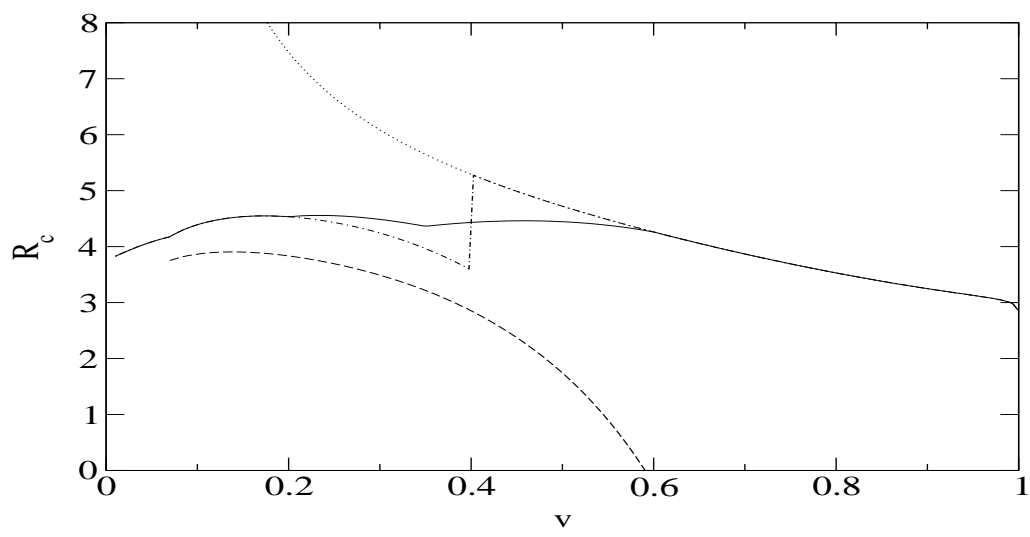

Figure 5: $\psi$-system: Solid line: interpolated spectral density; dashed-dotted line: reconstructed spectral density; dashed line: resummed spectral density; dotted line: perturbative spectral density.

smooth transition between the low and high energy region. We have chosen a quadratic form instead of a linear one since it suppresses better the behaviour of $R^{\text {Resum }}$ at high $v$ and of $R^{\text {Pert }}$ at low $v$. The moments from the interpolating spectral density are equal to the moments of the reconstructed spectral density at $v_{\text {sep }} \approx 0.4$, a typical nonrelativistic velocity. However, in the analysis we use the reconstructed spectral density and vary $v_{\text {sep }}$ between 0.3 and 0.5 to estimate the error.

In figure 6 we have shown the same picture, but now for typical scales of the upsilon system. Here the expansion behaves much better. $R^{\text {Resum }}$ is valid up to higher and $R^{\text {Pert }}$ to lower velocities. So we obtain an overlap in the intermediate region and the result is independent of variations around $v_{\text {sep }} \approx 0.35$.

\section{Numerical analysis for the bottom quark mass}

We now perform the analysis for the charm and bottom quark masses in the pole and PS-scheme. Though the method of analysis will be similar in all four cases we discuss every case separately as each requires a certain choice of parameters and an independent error analysis. Since in the upsilon system the theoretical expansions converge better we start with the bottom quark 


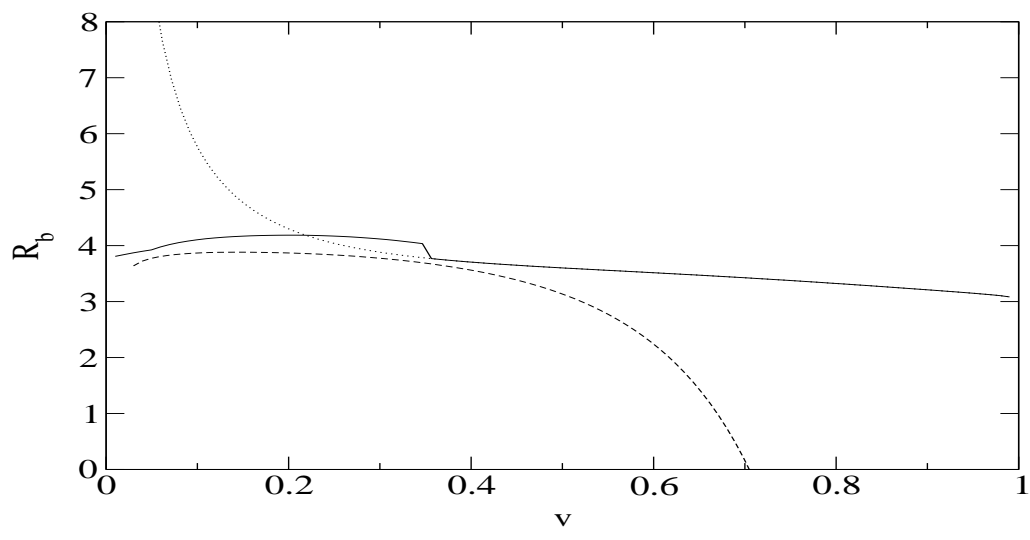

Figure 6: $\Upsilon$-system: Solid line: reconstructed spectral density; dashed line: resummed spectral density; dotted line: perturbative spectral density.

mass and devote the next section to the charm quark mass.

\subsection{Pole mass scheme}

First, one has to fix the parameters on which the sum rule depends. As a general rule, we will choose central values for the parameters to determine the masses and then vary these parameters in appropriate ranges for the error estimate. Let us start with the values of $\xi$ and $n$. Since the bottom quark is relatively heavy, even for $\xi=0$ the nonrelativistic and perturbative expansions converge reasonably well. Nevertheless, the contributions from the poles of the Green's function still dominate the theoretical part. To reduce their influence and to spread the theoretical contributions more equally among the poles, the resummed spectral density and the perturbative spectral density we must choose a higher $\xi$. However, for $\xi>1$ the moments loose sensitivity on the mass and the error from the input parameters increases. Therefore we use a central value of $\xi=0.5$ and vary $\xi$ between $0 \leq \xi \leq 1$. Since the relevant scale for the evaluation point is the lowest bound state energy, values of $\xi=0,0.5$ or 1 already correspond to well separated evaluation points. In the section on the PS-scheme we will justify this choice numerically as well.

As was shown in section 2, high values of $n$ enhance the threshold region and the pole contributions. To keep the theoretical expansions under control we restrict the moments to $n \leq 10$. From the lower side, $n$ is limited by the 


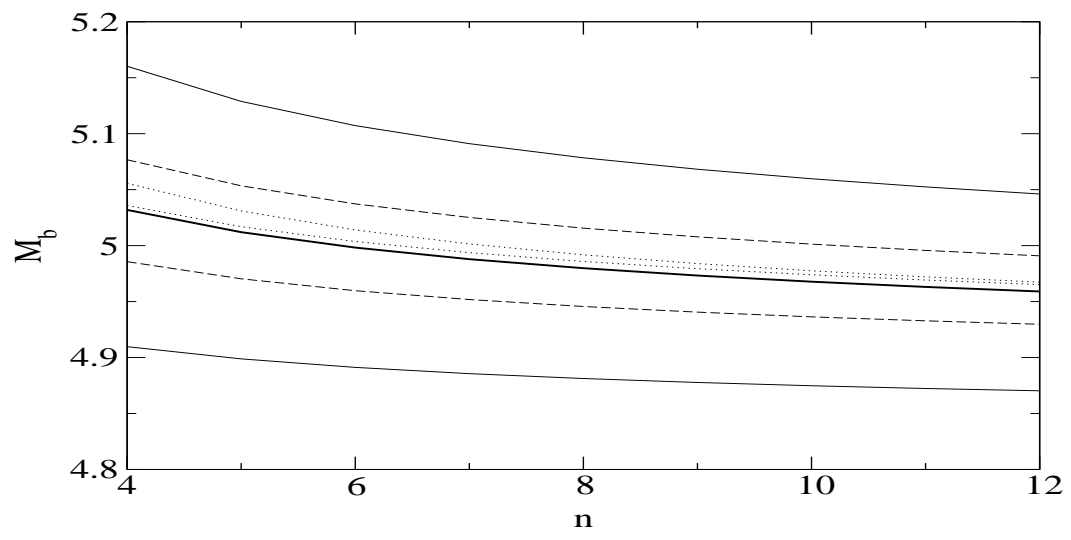

Figure 7: Thick solid line: central pole mass; thin solid lines: $M_{b}$ for $\mu_{\text {soft }}=$ 2.0 and $3.5 \mathrm{GeV}$; dashed lines: $M_{b}$ for $\mu_{f a c}=2.0$ and $5.0 \mathrm{GeV}$; dotted lines: $M_{b}$ for $\mu_{\text {hard }}=2.5$ and $10.0 \mathrm{GeV}$.

phenomenological uncertainty. Since for $n \leq 4$ the continuum has a large influence on the mass we use a range of $5 \leq n \leq 10$. As central values for our scales we have selected:

$$
\mu_{\text {soft }}=2.5 \mathrm{GeV}, \quad \mu_{\text {fac }}=3.5 \mathrm{GeV}, \quad \mu_{\text {hard }}=5.0 \mathrm{GeV} .
$$

We set the hard scale to the central value for the pole mass. The soft scale should be given by the mass times a typical velocity. We choose a value of $\mu_{\text {soft }}=2.5 \mathrm{GeV}$. Though one may prefer a slightly lower value of $\mu_{\text {soft }}$, the nonrelativistic expansion gets large corrections for $\mu_{\text {soft }}<2.0 \mathrm{GeV}$ and we will use this value as the lower bound in the variation of $\mu_{\text {soft }}$. The factorisation scale separates the different regions and should lie between the two other scales. The selected scales are required for a correct description of the spectral density. Since this is a physical quantity the scales must be chosen independent of $\xi$ and $n$ which merely serve as an evaluation point for the moments.

As discussed in the section about the phenomenological spectral density, we employ a continuum threshold of $\sqrt{s_{0}}=11.0 \pm 0.2 \mathrm{GeV}$. We use a separation velocity of $v_{\text {sep }}=0.35$ and the result is independent for a choice around this value. In the upsilon system the contribution from the condensates is suppressed by roughly a factor of $10^{-3}-10^{-4}$ as compared to the pole contributions and can be safely neglected.

In table 5 we have collected the individual moments for different val- 


\begin{tabular}{|c|c|c|c|c|c|c|}
\hline$n$ & 5 & 6 & 7 & 8 & 9 & 10 \\
\hline $\mathcal{M}_{n}^{\text {Poles }}$ & 0.079 & 0.055 & 0.038 & 0.026 & 0.018 & 0.013 \\
$\mathcal{M}_{n}^{\text {Rcstr }}$ & 0.098 & 0.055 & 0.032 & 0.019 & 0.011 & 0.0067 \\
$\mathcal{M}_{n}^{\text {Continuum }}$ & 0.047 & 0.023 & 0.012 & 0.0059 & 0.0031 & 0.0016 \\
\hline
\end{tabular}

Table 5: Moments for different $n$ with the parameters $\xi=0.5, \mu_{\text {soft }}=2.5$ $\mathrm{GeV}, \mu_{\text {fac }}=3.5 \mathrm{GeV}, \mu_{\text {hard }}=5.0 \mathrm{GeV}, \sqrt{s_{0}}=11.0 \mathrm{GeV}$ and $v_{\text {sep }}=0.35$.

ues of $n . \mathcal{M}_{n}^{\text {Poles }}$ are the theoretical poles of the Green's function. $\mathcal{M}_{n}^{\text {Rcstr }}$ contains the moments from the reconstructed spectral density and includes the moments from the resummed spectral density $\mathcal{M}_{n}^{\text {Resum }}$ below and the perturbative spectral density $\mathcal{M}_{n}^{\text {Pert }}$ above $v_{\text {sep }}$. There is no clear distinction between $\mathcal{M}_{n}^{\text {Resum }}$ and $\mathcal{M}_{n}^{\text {Pert }}$ since $v_{\text {sep }}$ can be used to shift their values. $\mathcal{M}_{n}^{\text {Continuum }}$ are the moments from the continuum part of the reconstructed spectral density above $s_{0}$. For values between $5 \leq n \leq 10$ the moments $\mathcal{M}_{n}^{R c s t r}$ are of a similar size as the pole contributions: for $n=5$ they exceed the poles and become smaller for $n \geq 7$. The influence of the continuum moments is relatively strong for small $n$ but gets more and more suppressed for higher $n$. The thick solid line in figure 7 shows the central value for the pole mass with the scales from eq. (41). Averaging over the mass between $5 \leq n \leq 10$ we obtain

$$
M_{b}=4.984 \mathrm{GeV} \text {. }
$$

The error originates mainly from the variation of the scales. For the soft scale we choose a range of $2.0 \mathrm{GeV} \leq \mu_{\text {soft }} \leq 3.5 \mathrm{GeV}$. Below $\mu_{\text {soft }}<2.0 \mathrm{GeV}$ the pole contributions show a bad perturbative behaviour and the analysis becomes unstable. $\mu_{\text {soft }}>3.5 \mathrm{GeV}$ would use a soft scale at an energy too high from physical expectation. The hard scale is varied between $M_{b} / 2$ and $2 M_{b}, 2.5 \mathrm{GeV} \leq \mu_{\text {hard }} \leq 10.0 \mathrm{GeV}$. As before for the central values, also the variation of the factorisation scale should lie between the two other scales and we use $2.0 \mathrm{GeV} \leq \mu_{f a c} \leq 5.0 \mathrm{GeV}$. In figure 7 we have also plotted the change of the mass for a variation of these scales. The error amounts to

$$
\begin{aligned}
2.0 \mathrm{GeV} \leq \mu_{\text {soft }} \leq 3.5 \mathrm{GeV}: & \Delta M_{b}=95 \mathrm{MeV} \\
2.0 \mathrm{GeV} \leq \mu_{\text {fac }} \leq 5.0 \mathrm{GeV}: & \Delta M_{b}=35 \mathrm{MeV} \\
2.5 \mathrm{GeV} \leq \mu_{\text {hard }} \leq 10.0 \mathrm{GeV}: & \Delta M_{b}=20 \mathrm{MeV}
\end{aligned}
$$

In table 6] we have listed the dependence of $\mathcal{M}_{7}^{\text {Poles }}$ and $\mathcal{M}_{7}^{R c s t r}$ on the scales. The soft scale has a particular large influence on the poles. In table [7 we have confronted the LO, NLO and NNLO corrections in NRQCD for $n=7$. Instead of the $\mathcal{M}_{7}^{\text {Rcstr }}$ we directly use $\mathcal{M}_{7}^{\text {Resum }}$ for this comparison. 


\begin{tabular}{|c|c|c|c|c|c|c|}
\hline$\mu_{\text {soft }}$ & 2.0 & 2.25 & 2.5 & 2.75 & 3.0 & 3.5 \\
\hline $\mathcal{M}_{7}^{\text {Poles }}$ & 0.050 & 0.043 & 0.038 & 0.034 & 0.032 & 0.028 \\
$\mathcal{M}_{7}^{\text {Rcstr }}$ & 0.034 & 0.032 & 0.032 & 0.031 & 0.031 & 0.030 \\
\hline \hline$\mu_{\text {fac }}$ & 2.0 & 3.0 & 3.5 & 4.0 & 4.5 & 5.0 \\
\hline $\mathcal{M}_{7}^{\text {Poles }}$ & 0.043 & 0.039 & 0.038 & 0.037 & 0.036 & 0.034 \\
$\mathcal{M}_{7}^{\text {Rcstr }}$ & 0.032 & 0.032 & 0.032 & 0.032 & 0.031 & 0.031 \\
\hline \hline$\mu_{\text {hard }}$ & 2.5 & 4.0 & 5.0 & 6.0 & 8.0 & 10.0 \\
\hline $\mathcal{M}_{7}^{\text {Poles }}$ & 0.038 & 0.038 & 0.038 & 0.038 & 0.039 & 0.039 \\
$\mathcal{M}_{7}^{\text {Rcstr }}$ & 0.035 & 0.032 & 0.032 & 0.031 & 0.031 & 0.030 \\
\hline
\end{tabular}

Table 6: $\mathcal{M}_{7}^{\text {Poles }}$ and $\mathcal{M}_{7}^{\text {Rcstr }}$ for different $\mu_{\text {soft }}$ with $\mu_{f a c}=3.5 \mathrm{GeV}$ and $\mu_{\text {hard }}=5.0 \mathrm{GeV}$, for different $\mu_{\text {fac }}$ with $\mu_{\text {soft }}=2.5 \mathrm{GeV}$ and $\mu_{\text {hard }}=5.0$ $\mathrm{GeV}$ and for different $\mu_{\text {hard }}$ with $\mu_{\text {soft }}=2.5 \mathrm{GeV}$ and $\mu_{\text {fac }}=3.5 \mathrm{GeV}$.

\begin{tabular}{|c|c|c|c|c|c|c|c|}
\hline$\mu_{\text {soft }}$ & & 2.0 & 2.25 & 2.5 & 2.75 & 3.0 & 3.5 \\
\hline \multirow{3}{*}{$\mathcal{M}_{7}^{\text {Poles }}$} & LO & 0.024 & 0.020 & 0.018 & 0.015 & 0.014 & 0.012 \\
& NLO & 0.033 & 0.029 & 0.027 & 0.024 & 0.022 & 0.019 \\
& NNLO & 0.050 & 0.043 & 0.038 & 0.034 & 0.032 & 0.028 \\
\hline \multirow{3}{*}{$\mathcal{M}_{7}^{\text {Resum }}$} & LO & 0.022 & 0.021 & 0.020 & 0.019 & 0.019 & 0.018 \\
& NLO & 0.014 & 0.014 & 0.014 & 0.014 & 0.014 & 0.014 \\
& NNLO & 0.016 & 0.015 & 0.014 & 0.014 & 0.014 & 0.013 \\
\hline
\end{tabular}

Table 7: Size of the moments from the poles and the resummed spectral density at LO, NLO and NNLO for different values of $\mu_{\text {soft }}$. 


\begin{tabular}{|l|c|}
\hline \multicolumn{1}{|c|}{ Source } & $\Delta M_{b}$ \\
\hline Variation of $\mu_{\text {soft }}$ & $95 \mathrm{MeV}$ \\
Variation of $\mu_{\text {fac }}$ & $35 \mathrm{MeV}$ \\
Variation of $\mu_{\text {hard }}$ & $20 \mathrm{MeV}$ \\
Threshold $s_{0}$ & $20 \mathrm{MeV}$ \\
Experimental widths & $30 \mathrm{MeV}$ \\
Variation of $\Lambda_{Q C D}$ & $50 \mathrm{MeV}$ \\
Variation of $\xi$ & $35 \mathrm{MeV}$ \\
\hline Total error & $125 \mathrm{MeV}$ \\
\hline
\end{tabular}

Table 8: Single contributions to the error of $M_{b}$.

The analysis confirms that the expansions converge better for smaller $n$ and behave worse for higher $n$ as is expected from the more sensitive testing of the threshold region. The corresponding shift of the mass by going from NLO to NNLO amounts to $\Delta M_{b} \approx 120 \mathrm{MeV}$.

Now we turn our attention to the other parameters. In the pole mass scheme, a significant uncertainty comes from $\Lambda_{Q C D}$ as well. To determine $\alpha_{s}(\mu)$ we have used $\alpha_{s}\left(M_{Z}\right)=0.1181 \pm 0.002$ [1] and run this value down with the three-loop beta function. The corresponding $\Lambda_{Q C D}$ for three loops and four massless flavours is then $\Lambda_{Q C D}=279 \pm 29 \mathrm{MeV}$. Though $\alpha_{s}$ is known relatively precise, the error on the mass amounts to $\Delta M_{b}=50 \mathrm{MeV}$ since the pole mass has a strong dependence on the coupling constant. The choice of the continuum threshold shifts the mass at low $n$ and gives an error of $\Delta M_{b}=20 \mathrm{MeV}$. The error from the experimentally measured decay widths is $\Delta M_{b}=30 \mathrm{MeV}$. When we employ a value of $\xi=0$ the mass decreases by $35 \mathrm{MeV}$ and rises by the same amount for $\xi=1$. We have summarised the results in table 8 . We have checked for the correlations between the errors from the different input parameters and have found almost no correlation between the errors. This holds also true for the PS-scheme and for the charm quark mass analysis. Thus we add the errors quadratically and our final result for the pole mass is

$$
M_{b}=4.984 \pm 0.125 \mathrm{GeV} .
$$

Using the three-loop relation between the pole and the $\overline{\mathrm{MS}}$-mass which has been calculated recently [41, 42, we obtain $m_{b}\left(m_{b}\right)=4.277 \pm 0.116 \mathrm{GeV}$ for the $\overline{\mathrm{MS}}$-mass. However, the relation between the two masses implicitly includes an uncertainty of $O\left(\Lambda_{Q C D}\right)$.

Before turning to the analysis in the PS-scheme we want to investigate the size of the NNNLO-corrections from NRQCD. In [43] the leading-log 
term of $O\left(\alpha_{s}^{3} \ln \alpha_{s}\right)$ to the energy levels has been derived in the framework of potential NRQCD. The results have been confirmed in 44] where also the corrections to the wave function at $O\left(\alpha_{s}^{3} \ln ^{2} \alpha_{s}\right)$ were calculated. Recently, the full NNNLO corrections have been computed [45, 46, but only for the energy levels itself. The results of [44] can be used to estimate the impact on the Green's function and the mass. The main contributions come from the two lowest bound states. With a typical soft scale of $\mu_{\text {soft }}=2.5 \mathrm{GeV}$ for the Green's function, the $O\left(\alpha_{s}^{3}\right)$-contributions lower the mass by approximately $35 \mathrm{MeV}, \Delta M_{b}=-35 \mathrm{MeV}$. We have not included this mass shift in our final result since these contributions represent only a part of the full NNNLO corrections. Furthermore, the results have only been derived for $\left|\psi_{n}(0)\right|^{2}$ and $E_{n}$ and not for the Green's function itself. Since the expansion for the energy and the wave function is not very good, the mass shift may be overestimated. However, it could indicate the size of the NNNLO corrections.

\subsection{Potential-subtracted mass scheme}

Here the separation scale $\mu_{\text {sep }}$ appears as an additional parameter which enters in the definition of the PS-mass (32). This scale should be taken large enough in order to guarantee a perturbative relation to the $\overline{\mathrm{MS}}$-mass. On the other hand, it should be smaller than $M v$ as not to affect the threshold behaviour. A good value is $\mu_{\text {sep }}=2.0 \mathrm{GeV}$ and we will investigate a range of $1.0 \mathrm{GeV} \leq \mu_{\text {sep }} \leq 3.0 \mathrm{GeV}$ to check the influence on the $\overline{\mathrm{MS}}$-mass. In figure 8 we have plotted the PS-mass as a function of $n$. As our central values we obtain

$$
m_{P S, b}\left(\mu_{s e p}=2 \mathrm{GeV}\right)=4.561 \mathrm{GeV}, \quad m_{b}\left(m_{b}\right)=4.241 \mathrm{GeV}
$$

Relating the pole, PS- and $\overline{\mathrm{MS}}$-masses we make use of the recently calculated three-loop result for the masses 4142. Since resummation includes all orders in $\alpha_{s}$, this choice is more appropriate than the two-loop relation. From the variations of the scales we obtain

$$
\begin{aligned}
2.0 \mathrm{GeV} \leq \mu_{\text {soft }} \leq 3.5 \mathrm{GeV}: & \Delta m_{P S, b}=90 \mathrm{MeV} \\
2.0 \mathrm{GeV} \leq \mu_{\text {fac }} \leq 5.0 \mathrm{GeV}: & \Delta m_{P S, b}=35 \mathrm{MeV} \\
2.5 \mathrm{GeV} \leq \mu_{\text {hard }} \leq 10.0 \mathrm{GeV}: & \Delta m_{P S, b}=10 \mathrm{MeV}
\end{aligned}
$$

In table 9 we again have collected the moments for different values of $n$. For low $n, \mathcal{M}_{n}^{\text {Rcstr }}$ exceeds $\mathcal{M}_{n}^{\text {Poles }}$ whose influence grows for larger $n$. In tables [10 and 11] we have shown the scale dependence and the behaviour for the different orders. The large coefficients which have been found in the Coulomb 


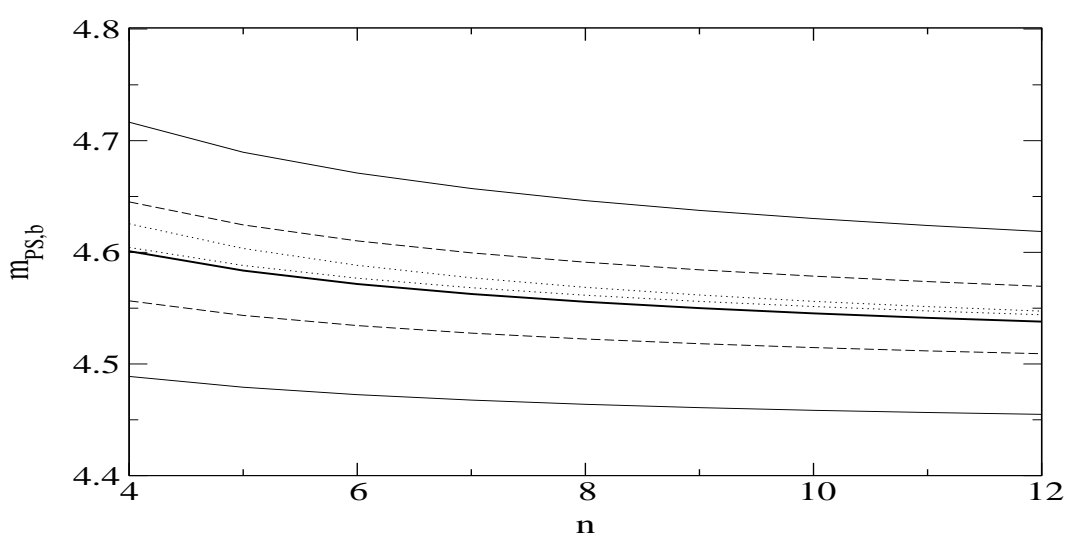

Figure 8: Thick solid line: central PS-mass; thin solid lines: $m_{P S, b}$ for $\mu_{\text {soft }}=2.0$ and $3.5 \mathrm{GeV}$; dashed lines: $m_{P S, b}$ for $\mu_{f a c}=2.0$ and $5.0 \mathrm{GeV}$; dotted lines: $m_{P S, b}$ for $\mu_{\text {hard }}=2.5$ and $10.0 \mathrm{GeV}$.

\begin{tabular}{|c|c|c|c|c|c|c|}
\hline$n$ & 5 & 6 & 7 & 8 & 9 & 10 \\
\hline $\mathcal{M}_{n}^{\text {Poles }}$ & 0.043 & 0.027 & 0.017 & 0.010 & 0.0063 & 0.0039 \\
$\mathcal{M}_{n}^{\text {Restr }}$ & 0.056 & 0.028 & 0.014 & 0.0076 & 0.0040 & 0.0022 \\
$\mathcal{M}_{n}^{\text {Continuum }}$ & 0.025 & 0.011 & 0.0047 & 0.0021 & 0.00095 & 0.00044 \\
\hline
\end{tabular}

Table 9: Moments for different $n$ with the parameters $\mu_{\text {soft }}=2.5 \mathrm{GeV}$, $\mu_{\text {fac }}=3.5 \mathrm{GeV}, \mu_{\text {hard }}=5.0 \mathrm{GeV}, \sqrt{s_{0}}=11.0 \mathrm{GeV}$ and $v_{\text {sep }}=0.35$.

\begin{tabular}{|c|c|c|c|c|c|c|}
\hline$\mu_{\text {soft }}$ & 2.0 & 2.25 & 2.5 & 2.75 & 3.0 & 3.5 \\
\hline $\mathcal{M}_{7}^{\text {Poles }}$ & 0.022 & 0.019 & 0.017 & 0.015 & 0.014 & 0.012 \\
$\mathcal{M}_{7}^{\text {Rcstr }}$ & 0.016 & 0.015 & 0.014 & 0.014 & 0.014 & 0.014 \\
\hline \hline$\mu_{\text {fac }}$ & 2.0 & 3.0 & 3.5 & 4.0 & 4.5 & 5.0 \\
\hline $\mathcal{M}_{7}^{\text {Poles }}$ & 0.019 & 0.017 & 0.017 & 0.016 & 0.016 & 0.015 \\
$\mathcal{M}_{7}^{\text {Rcstr }}$ & 0.015 & 0.015 & 0.014 & 0.014 & 0.014 & 0.014 \\
\hline \hline$\mu_{\text {hard }}$ & 2.5 & 4.0 & 5.0 & 6.0 & 8.0 & 10.0 \\
\hline $\mathcal{M}_{7}^{\text {Poles }}$ & 0.017 & 0.017 & 0.017 & 0.017 & 0.017 & 0.017 \\
$\mathcal{M}_{7}^{\text {Rcstr }}$ & 0.016 & 0.015 & 0.014 & 0.014 & 0.014 & 0.014 \\
\hline \hline
\end{tabular}

Table 10: $\mathcal{M}_{7}^{\text {Poles }}$ and $\mathcal{M}_{7}^{\text {Rcstr }}$ for different $\mu_{\text {soft }}$ with $\mu_{\text {fac }}=3.5 \mathrm{GeV}$ and $\mu_{\text {hard }}=5.0 \mathrm{GeV}$, for different $\mu_{\text {fac }}$ with $\mu_{\text {soft }}=2.5 \mathrm{GeV}$ and $\mu_{\text {hard }}=5.0$ $\mathrm{GeV}$ and for different $\mu_{\text {hard }}$ with $\mu_{\text {soft }}=2.5 \mathrm{GeV}$ and $\mu_{\text {fac }}=3.5 \mathrm{GeV}$. 


\begin{tabular}{|c|c|c|c|c|c|c|c|}
\hline$\mu_{\text {soft }}$ & & 2.0 & 2.25 & 2.5 & 2.75 & 3.0 & 3.5 \\
\hline \multirow{3}{*}{$\mathcal{M}_{7}^{\text {Poles }}$} & LO & 0.011 & 0.0089 & 0.0077 & 0.0067 & 0.0061 & 0.0051 \\
& NLO & 0.015 & 0.013 & 0.012 & 0.011 & 0.0097 & 0.0084 \\
& NNLO & 0.022 & 0.019 & 0.017 & 0.015 & 0.014 & 0.012 \\
\hline \multirow{3}{*}{$\mathcal{M}_{7}^{\text {Resum }}$} & LO & 0.010 & 0.0098 & 0.0094 & 0.0090 & 0.0087 & 0.0082 \\
& NLO & 0.0070 & 0.0070 & 0.0070 & 0.0070 & 0.0069 & 0.0068 \\
& NNLO & 0.0077 & 0.0072 & 0.0068 & 0.0066 & 0.0064 & 0.0062 \\
\hline
\end{tabular}

Table 11: Size of the moments from the poles and the resummed spectral density at LO, NLO and NNLO for different values of $\mu_{\text {soft }}$.

potential at NNLO (158) lead to a mass shift of $\Delta m_{b}\left(m_{b}\right) \approx 90-100 \mathrm{MeV}$ when going from NLO to NNLO.

When we lower the continuum threshold to $\sqrt{s_{0}}=10.8 \mathrm{GeV}$, the mass decreases for small $n$. For $\sqrt{s_{0}}=10.7 \mathrm{GeV}$, the mass is completely stable for all values of $3 \leq n \leq 12$. But since the analysis is too complex to draw conclusions on single parameters, we will not use the argument of stability to fix the values of the parameters. For higher values of $\sqrt{s_{0}}=11.2 \mathrm{GeV}$ the mass increases for small $n$. The error from this variation is $\Delta m_{P S, b}=15 \mathrm{MeV}$. Assuming that the continuum from open B-production can be neglected, $\sqrt{s_{0}}$ should lie above the highest resonance. Keeping the threshold at $\sqrt{s_{0}}=11.0$ $\mathrm{GeV}$, we must remove the 6 th resonance. Then the mass increases by $6 \mathrm{MeV}$. If we assume a very high threshold and also remove the 5th resonance the mass rises by $22 \mathrm{MeV}$. The error from the experimental decay widths is of a similar size, $\Delta m_{P S, b}=25 \mathrm{MeV}$. The main part comes from the width of the first resonance though it is not as dominant as in the charmonium system. In the PS-scheme, the influence of $\alpha_{s}$ is significantly reduced. Using the same $\Lambda_{Q C D}$ as before, we obtain $\Delta m_{P S, b}=20 \mathrm{MeV}$ for the PS-mass and $\Delta m_{b}\left(m_{b}\right)=5 \mathrm{MeV}$ for the $\overline{\mathrm{MS}}$-mass. From this weak dependence on the strong coupling constant we can not use the analysis for an estimate of $\alpha_{s}$.

Besides the contributions from resummation, this analysis includes the perturbative spectral density above $v_{\text {sep }}$ as well. Therefore it is interesting to investigate the influence of the perturbative part on the analysis. In figure 9 we have depicted the central mass with a solid line. Then we keep all contributions, including the poles, the resummed spectral density and the phenomenological part unchanged except for the perturbative spectral density for which we use only the lowest order. The resulting mass is shown as a dashed line. The analysis becomes unstable for low $n$ since here the perturbative contributions play an important part. Now we remove the perturbative part completely and use only the resummed spectral density. The 


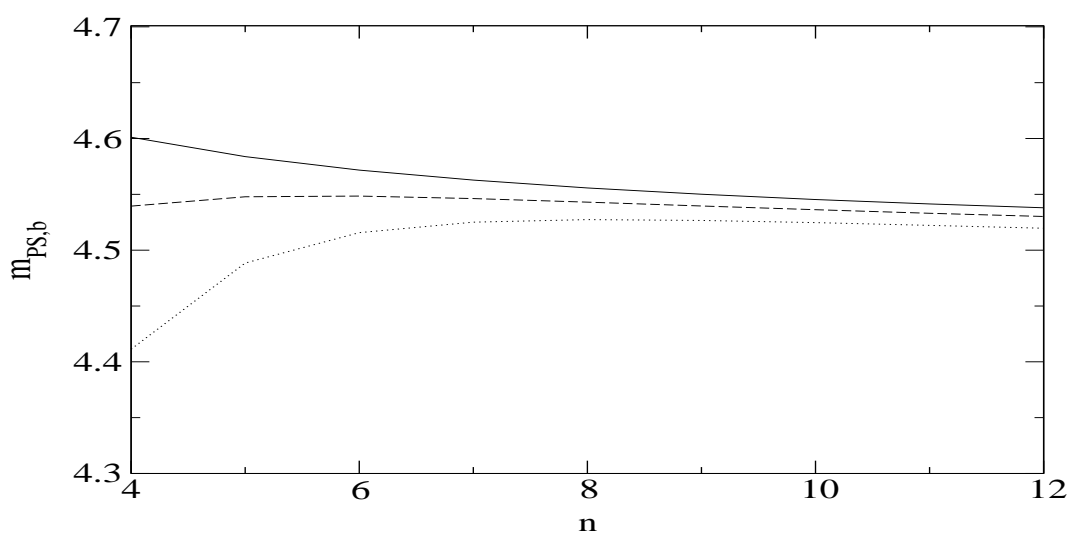

Figure 9: Solid line: central PS-mass; dashed line: perturbative contribution only at LO; dotted line: without perturbative contribution.

\begin{tabular}{|c|c|c|c|c|c|}
\hline$\mu_{s e p}$ & 1.0 & 1.5 & 2.0 & 2.5 & 3.0 \\
\hline$m_{P S, b}$ & 4.710 & 4.631 & 4.561 & 4.497 & 4.438 \\
$m_{b}\left(m_{b}\right)$ & 4.234 & 4.237 & 4.241 & 4.245 & 4.248 \\
\hline
\end{tabular}

Table 12: Change of the masses for different values of $\mu_{\text {sep }}$.

dotted line signals clearly that essential information for low $n$ is lost. Only for high $n$, dominated by the poles, the analysis becomes more stable but here also the expansion of the poles behaves more badly.

In table 12 we have varied the separation scale $\mu_{\text {sep }}$ between $1.0 \mathrm{GeV} \leq$ $\mu_{\text {sep }} \leq 3.0 \mathrm{GeV}$. The stability of the PS-mass as a function of $n$ remains almost unchanged, but the value of the PS-mass changes strongly as its definition depends directly on $\mu_{\text {sep }}$. In the relation to the $\overline{\mathrm{MS}}$-mass this variation is cancelled to such an extend that the $\overline{\mathrm{MS}}$-mass changes only by 7 $\mathrm{MeV}$.

Now we want to discuss the choice of $\xi$ in more detail. Using a higher $\xi$ the theoretical expansions converge better and the dominance of the pole contributions is reduced. As a result, the theoretical moments are more equally distributed. Thus the dependence on a single contribution like the poles is reduced. In this way one gets a better control over the systematic uncertainties in the sum rules. Table 13 shows the $\overline{M S}$-mass for different $\xi$. As a measure of the uncertainty we now investigate the change of the mass connected with the expansion of the poles in NRQCD. The first entry 


\begin{tabular}{|c|c|c|c|}
\hline$\xi$ & $m_{b}\left(m_{b}\right) /[\mathrm{GeV}]$ & $\Delta m_{b}^{(a)} /[\mathrm{MeV}]$ & $\Delta m_{b}^{(b)} /[\mathrm{MeV}]$ \\
\hline-0.25 & 4.200 & 71 & 114 \\
0 & 4.215 & 62 & 102 \\
0.5 & 4.241 & 54 & 90 \\
1.0 & 4.262 & 49 & 82 \\
1.5 & 4.278 & 46 & 79 \\
2.0 & 4.292 & 45 & 77 \\
\hline
\end{tabular}

Table 13: Change of the $\overline{\mathrm{MS}}$-mass, when adding the difference from NNLONLO to the poles, $\Delta m_{b}^{(a)}$, and the difference from NNLO-LO, $\Delta m_{b}^{(b)}$.

\begin{tabular}{|l|c|r|}
\hline \multicolumn{1}{|c|}{ Source } & $\Delta m_{P S, b}$ & $\Delta m_{b}\left(m_{b}\right)$ \\
\hline Variation of $\mu_{\text {soft }}$ & $90 \mathrm{MeV}$ & $80 \mathrm{MeV}$ \\
Variation of $\mu_{\text {fac }}$ & $35 \mathrm{MeV}$ & $30 \mathrm{MeV}$ \\
Variation of $\mu_{\text {hard }}$ & $10 \mathrm{MeV}$ & $10 \mathrm{MeV}$ \\
Variation of $\mu_{\text {sep }}$ & - & $5 \mathrm{MeV}$ \\
Threshold $s_{0}$ & $25 \mathrm{MeV}$ & $25 \mathrm{MeV}$ \\
Experimental error & $35 \mathrm{MeV}$ & $30 \mathrm{MeV}$ \\
Variation of $\Lambda_{Q C D}$ & $20 \mathrm{MeV}$ & $5 \mathrm{MeV}$ \\
Variation of $\xi$ & $30 \mathrm{MeV}$ & $25 \mathrm{MeV}$ \\
\hline Total error & $112 \mathrm{MeV}$ & $98 \mathrm{MeV}$ \\
\hline
\end{tabular}

Table 14: Single contributions to the error of $m_{P S, b}$ and $m_{b}\left(m_{b}\right)$.

shows the central value for the mass. Now we add to the poles of the Green's function the difference between the NNLO and the NLO result. The increase of the mass is shown in the second column. In the third column we add the difference between the NNLO and the LO result. Here the error on the mass from the expansion of the poles decreases for higher $\xi$. If we assume this as a conservative error estimate, for $\xi=0.5$ this error is of the same order as the error from the variation of the soft scale. In fact, the error from the scales increases for higher $\xi$ as a result of the decreasing sensibility on the mass. From the viewpoint of the convergence of the series, the scale variation tends to underestimate the error for low $\xi$ and to overestimate the error for higher $\xi$. However, for $\xi=0.5$ both estimates are consistent with each other and we have thus chosen this value as our default. For $\xi \gtrsim 1$ the better control over the theoretical expansions is not large enough to compensate for the decreasing sensibility on the mass and the increasing influence of the other contributions. The error from $0 \leq \xi \leq 1$ is $\Delta m_{P S, b}=30 \mathrm{MeV}$. Table 
14 summarises the error from all contributions for $m_{P S, b}$ and $m_{b}\left(m_{b}\right)$. Our results are

$$
\begin{aligned}
m_{P S, b}\left(\mu_{\text {sep }}=2.0\right) & =4.561 \pm 0.112 \mathrm{GeV} \\
m_{b}\left(m_{b}\right) & =4.241 \pm 0.098 \mathrm{GeV} .
\end{aligned}
$$

This value can be compared to the $\overline{\mathrm{MS}}$-mass obtained from the pole mass scheme, $m_{b}\left(m_{b}\right)=4.277 \pm 0.116 \mathrm{GeV}$. The central value decreases by $30 \mathrm{MeV}$. In the PS-scheme one has better control over the systematic uncertainties, reflected in an improved convergence for the theoretical expansions and in clear perturbative mass relation.

\section{$9 \quad$ Numerical analysis for the charm quark mass}

\subsection{Pole mass scheme}

The method of analysis will follow along the same lines as for the bottom quark mass and we will put special emphasis on the points different in both analyses. First we must choose the value of $\xi$. As for the bottomium, we will use $\xi=0.5$. At this value the pole contributions still represent the dominant part. In principle one would like to choose a higher value where the theoretical expansions converge better. However, the contribution from the theoretical poles varies significantly with the scales; for $\xi \gtrsim 1$ the mass depends too strongly on these variations. Thus we again use a range of $0 \leq \xi \leq 1$. Again, in the PS-scheme we will investigate the perturbative behaviour for different $\xi$ in more detail. Since the perturbative expansions converge more slowly than for the upsilon we restrict the analysis to smaller values of $n \leq 7$. From the lower side, we choose $n \geq 4$ since for $\xi=0.5$ the moments at $n=3$ already depend significantly on the phenomenological part. As central values for our scales we have selected:

$$
\mu_{\text {soft }}=1.2 \mathrm{GeV}, \quad \mu_{\text {fac }}=1.45 \mathrm{GeV}, \quad \mu_{\text {hard }}=1.75 \mathrm{GeV}
$$

The hard scale corresponds to the central value of the pole mass. For the soft scale we would have preferred a somewhat smaller value but then the NNLO corrections become large. The moments for different values of $n$ are shown in table 15. $\mathcal{M}_{n}^{\text {Rcstr }}$ are the moments from the reconstructed spectral density at

$v_{\text {sep }}=0.4$. At this separation velocity $\mathcal{M}_{n}^{\text {Rcstr }}$ equal the moments from the interpolating spectral density which was introduced in section 7 . The pole contributions dominate the sum rule even for small $n$. The condensates are suppressed compared to the poles and have no influence on the mass. From 


\begin{tabular}{|c|c|c|c|c|c|c|}
\hline$n$ & 3 & 4 & 5 & 6 & 7 & 8 \\
\hline $\mathcal{M}_{n}^{\text {Poles }}$ & 0.65 & 0.48 & 0.35 & 0.26 & 0.19 & 0.14 \\
$\mathcal{M}_{n}^{\text {Rcstr }}$ & 0.41 & 0.21 & 0.11 & 0.063 & 0.036 & 0.021 \\
$\mathcal{M}_{n}^{\text {Continuum }}$ & 0.23 & 0.099 & 0.046 & 0.023 & 0.011 & 0.0058 \\
$\mathcal{M}_{n}^{\text {Condensates }}$ & -0.0033 & -0.0030 & -0.0027 & -0.0023 & -0.0019 & -0.0015 \\
\hline
\end{tabular}

Table 15: Moments for different $n$ with the parameters $\mu_{\text {soft }}=1.2 \mathrm{GeV}$, $\mu_{\text {fac }}=1.45 \mathrm{GeV}, \mu_{\text {hard }}=1.75 \mathrm{GeV}$ and $v_{\text {sep }}=0.4$.

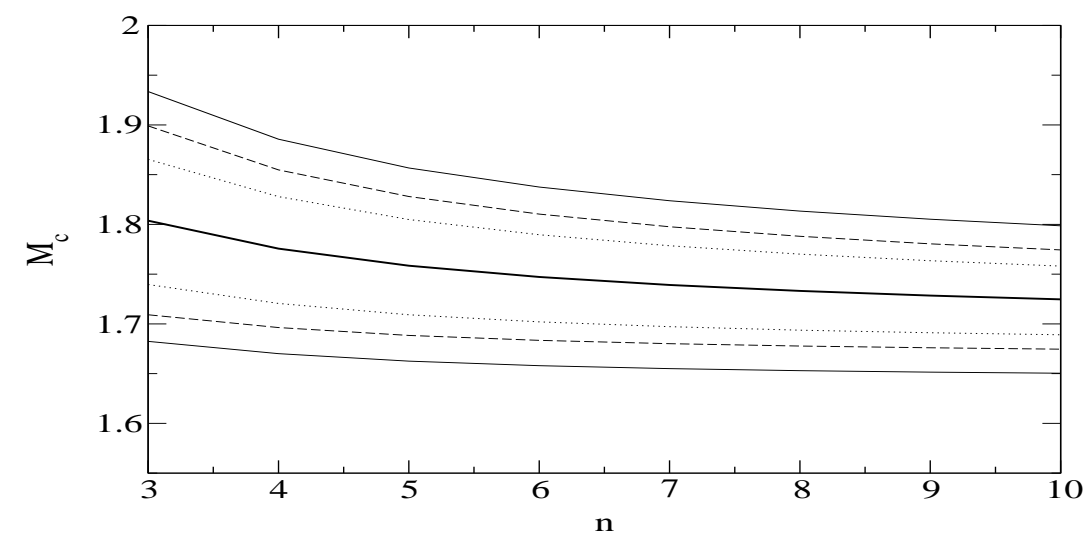

Figure 10: Thick solid line: central pole mass; thin solid lines: $M_{c}$ for $\mu_{\text {soft }}=1.1$ and $1.35 \mathrm{GeV}$; dashed lines: $M_{c}$ for $\mu_{\text {fac }}=1.2$ and $1.65 \mathrm{GeV}$; dotted lines: $M_{c}$ for $\mu_{\text {hard }}=1.4$ and $2.5 \mathrm{GeV}$. 


\begin{tabular}{|c|c|c|c|c|c|c|}
\hline$\mu_{\text {soft }}$ & 1.1 & 1.15 & 1.2 & 1.25 & 1.3 & 1.35 \\
\hline $\mathcal{M}_{5}^{\text {Poles }}$ & 0.51 & 0.42 & 0.36 & 0.31 & 0.27 & 0.24 \\
$\mathcal{M}_{5}^{\text {Rcstr }}$ & 0.12 & 0.11 & 0.11 & 0.11 & 0.10 & 0.10 \\
\hline \hline$\mu_{\text {fac }}$ & 1.2 & 1.3 & 1.4 & 1.5 & 1.6 & 1.7 \\
\hline $\mathcal{M}_{5}^{\text {Poles }}$ & 0.46 & 0.42 & 0.38 & 0.34 & 0.29 & 0.25 \\
$\mathcal{M}_{5}^{\text {Rcstr }}$ & 0.12 & 0.11 & 0.11 & 0.11 & 0.10 & 0.096 \\
\hline \hline$\mu_{\text {hard }}$ & 1.4 & 1.6 & 1.8 & 2.0 & 2.2 & 2.5 \\
\hline $\mathcal{M}_{5}^{\text {Poles }}$ & 0.29 & 0.33 & 0.36 & 0.39 & 0.41 & 0.43 \\
$\mathcal{M}_{5}^{\text {Rcstr }}$ & 0.11 & 0.11 & 0.11 & 0.11 & 0.11 & 0.11 \\
\hline
\end{tabular}

Table 16: $\mathcal{M}_{5}^{\text {Poles }}$ and $\mathcal{M}_{5}^{\text {Rcstr }}$ for different $\mu_{\text {soft }}$ with $\mu_{\text {fac }}=1.45 \mathrm{GeV}$ and $\mu_{\text {hard }}=1.75 \mathrm{GeV}$, for different $\mu_{\text {fac }}$ with $\mu_{\text {soft }}=1.2 \mathrm{GeV}$ and $\mu_{\text {hard }}=1.75$ $\mathrm{GeV}$ and for different $\mu_{\text {hard }}$ with $\mu_{\text {soft }}=1.2 \mathrm{GeV}$ and $\mu_{\text {fac }}=1.45 \mathrm{GeV}$.

figure 10, averaging over $4 \leq n \leq 7$, we obtain

$$
M_{c}=1.754 \mathrm{GeV} \text {. }
$$

The error is dominated by the variation of the scales. For values of $\mu_{\text {soft }} \lesssim$ 1.1 GeV the pole contributions get large NNLO corrections and we thus choose $1.1 \mathrm{GeV} \leq \mu_{\text {soft }} \leq 1.35 \mathrm{GeV}$. For the hard scale we use a range of $1.4 \mathrm{GeV} \leq \mu_{\text {hard }} \leq 2.5 \mathrm{GeV}$ and for the factorisation scale $1.2 \mathrm{GeV} \leq \mu_{\text {fac }} \leq$ $1.65 \mathrm{GeV}$. Since the convergence of the nonrelativistic expansion is not very good for the charmonium system, the scales cannot be chosen arbitrarily far away from their central values. Though the analysis is stable inside the given intervals, the expressions tend to become unstable for scales outside of the chosen ranges. The error amounts to

$$
\begin{aligned}
1.1 \mathrm{GeV} \leq \mu_{\text {soft }} \leq 1.35 \mathrm{GeV}: & \Delta M_{c}=90 \mathrm{MeV}, \\
1.2 \mathrm{GeV} \leq \mu_{\text {fac }} \leq 1.65 \mathrm{GeV}: & \Delta M_{c}=65 \mathrm{MeV}, \\
1.4 \mathrm{GeV} \leq \mu_{\text {hard }} \leq 2.5 \mathrm{GeV}: & \Delta M_{c}=40 \mathrm{MeV}
\end{aligned}
$$

Tables [16] and 17 show the dependence of $\mathcal{M}_{5}^{\text {Poles }}$ and $\mathcal{M}_{5}^{\text {Rcstr }}$ on the scales and of $\mathcal{M}_{5}^{\text {Poles }}$ and $\mathcal{M}_{5}^{\text {Resum }}$ from the different orders on $\mu_{\text {soft }}$ respectively.

To estimate the uncertainty from $\alpha_{s}$ we employ $\Lambda_{Q C D}=329 \pm 29 \mathrm{MeV}$ which is the corresponding value for 3 flavours and 3 loops. Then the mass shifts by $60 \mathrm{MeV}$. Since already the lowest resonances dominate the phenomenological part, the error from the measured spectral density and experimental widths is relatively small. For $\xi=0$ the mass decreases by $60 \mathrm{MeV}$ and increases by the same amount for $\xi=1$. From table 18 we then obtain 


\begin{tabular}{|c|c|c|c|c|c|c|c|}
\hline$\mu_{\text {soft }}$ & & 1.1 & 1.15 & 1.2 & 1.25 & 1.3 & 1.35 \\
\hline \multirow{3}{*}{$\mathcal{M}_{5}^{\text {Poles }}$} & LO & 0.19 & 0.17 & 0.15 & 0.13 & 0.12 & 0.11 \\
& NLO & 0.31 & 0.28 & 0.25 & 0.23 & 0.21 & 0.19 \\
& NNLO & 0.51 & 0.42 & 0.36 & 0.31 & 0.27 & 0.24 \\
\hline \multirow{3}{*}{$\mathcal{M}_{5}^{\text {Resum }}$} & LO & 0.097 & 0.093 & 0.090 & 0.087 & 0.085 & 0.083 \\
& NLO & 0.043 & 0.046 & 0.047 & 0.048 & 0.049 & 0.050 \\
& NNLO & 0.045 & 0.040 & 0.036 & 0.033 & 0.031 & 0.029 \\
\hline
\end{tabular}

Table 17: Size of the moments from the poles and the resummed spectral density at LO, NLO and NNLO for different values of $\mu_{\text {soft }}$.

\begin{tabular}{|l|r|}
\hline \multicolumn{1}{|c|}{ Source } & \multicolumn{1}{|c|}{$\Delta M_{c}$} \\
\hline Variation of $\mu_{\text {soft }}$ & $90 \mathrm{MeV}$ \\
Variation of $\mu_{\text {fac }}$ & $65 \mathrm{MeV}$ \\
Variation of $\mu_{\text {hard }}$ & $40 \mathrm{MeV}$ \\
Experimental cross section & $5 \mathrm{MeV}$ \\
Experimental widths & $20 \mathrm{MeV}$ \\
Variation of $v_{\text {sep }}$ & $10 \mathrm{MeV}$ \\
Variation of $\Lambda_{Q C D}$ & $60 \mathrm{MeV}$ \\
Variation of $\xi$ & $60 \mathrm{MeV}$ \\
\hline Total error & $147 \mathrm{MeV}$ \\
\hline
\end{tabular}

Table 18: Single contributions to the error of $M_{c}$.

the pole mass

$$
M_{c}=1.754 \pm 0.147 \mathrm{GeV} .
$$

This corresponds to a $\overline{\mathrm{MS}}$-mass of $m_{c}\left(m_{c}\right)=1.247 \pm 0.134 \mathrm{GeV}$. Again, there is an $O\left(\Lambda_{Q C D}\right)$ uncertainty from the perturbative relation between the masses so we now turn to the PS-scheme to determine the $\overline{\mathrm{MS}}$-mass.

\subsection{Potential-subtracted mass scheme}

As in the pole scheme, we will use $\xi=0.5$ within a range of $0 \leq \xi \leq 1$. For the separation scale we choose $\mu_{\text {sep }}=1.0 \pm 0.2 \mathrm{GeV}$. This represents a compromise value. It is still high enough for a perturbative evaluation and sufficiently below the hard scale. Since in the PS-scheme the theoretical expansions converge better, one can employ a lower value for the soft scale and we will use $\mu_{\text {soft }}=1.1 \mathrm{GeV}$. As before, we use a range of $4 \leq n \leq 7$ for the moments. 


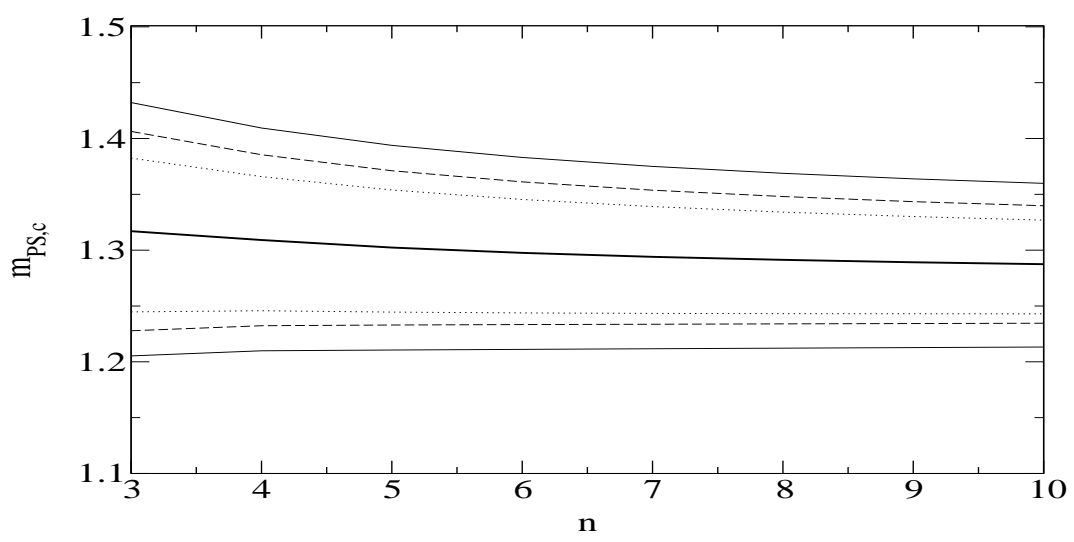

Figure 11: Thick solid line: central PS-mass; thin solid lines: $m_{P S, c}$ for $\mu_{\text {soft }}=1.0$ and $1.25 \mathrm{GeV}$; dashed lines: $m_{P S, c}$ for $\mu_{f a c}=1.2$ and $1.65 \mathrm{GeV}$; dotted lines: $m_{P S, c}$ for $\mu_{\text {hard }}=1.4$ and $2.5 \mathrm{GeV}$.

\begin{tabular}{|c|c|c|c|c|c|c|}
\hline$n$ & 3 & 4 & 5 & 6 & 7 & 8 \\
\hline $\mathcal{M}_{n}^{\text {Poles }}$ & 0.22 & 0.11 & 0.056 & 0.028 & 0.014 & 0.0071 \\
$\mathcal{M}_{n}^{\text {Rcstr }}$ & 0.13 & 0.043 & 0.016 & 0.0059 & 0.0022 & 0.00088 \\
$\mathcal{M}_{n}^{\text {Continuum }}$ & 0.057 & 0.016 & 0.0047 & 0.0015 & 0.00047 & 0.00015 \\
$\mathcal{M}_{n}^{\text {Condensates }}$ & -0.0016 & -0.00097 & -0.00057 & -0.00032 & -0.00018 & -0.000096 \\
\hline
\end{tabular}

Table 19: Moments for different $n$ with the parameters $\mu_{\text {soft }}=1.1 \mathrm{GeV}$, $\mu_{\text {fac }}=1.45 \mathrm{GeV}, \mu_{\text {hard }}=1.75 \mathrm{GeV}$ and $v_{\text {sep }}=0.4$.

In table 19] we have shown the moments for different $n$. The poles represent the dominant part of the theoretical correlator. The size of the condensates is $\approx 1 \%$ of the pole contributions and also in this scheme they can be neglected for the analysis.

Our central values for the PS- and $\overline{\mathrm{MS}}$-masses are

$$
m_{P S, c}\left(\mu_{\text {sep }}=1 \mathrm{GeV}\right)=1.300 \mathrm{GeV}, \quad m_{c}\left(m_{c}\right)=1.188 \mathrm{GeV} .
$$

In figure 11] we have plotted the PS-mass and the corresponding error from the scales:

$$
\begin{array}{ll}
1.0 \mathrm{GeV} \leq \mu_{\text {soft }} \leq 1.25 \mathrm{GeV}: & \Delta m_{P S, c}=85 \mathrm{MeV}, \\
1.2 \mathrm{GeV} \leq \mu_{\text {fac }} \leq 1.65 \mathrm{GeV}: & \Delta m_{P S, c}=65 \mathrm{MeV},
\end{array}
$$




\begin{tabular}{|c|c|c|c|c|c|c|}
\hline$\mu_{\text {soft }}$ & 1.0 & 1.05 & 1.1 & 1.15 & 1.2 & 1.25 \\
\hline $\mathcal{M}_{5}^{\text {Poles }}$ & 0.10 & 0.075 & 0.057 & 0.045 & 0.036 & 0.030 \\
$\mathcal{M}_{5}^{\text {Rcstr }}$ & 0.020 & 0.017 & 0.015 & 0.013 & 0.012 & 0.011 \\
\hline \hline$\mu_{\text {fac }}$ & 1.2 & 1.3 & 1.4 & 1.5 & 1.6 & 1.7 \\
\hline $\mathcal{M}_{5}^{\text {Poles }}$ & 0.083 & 0.072 & 0.062 & 0.052 & 0.043 & 0.034 \\
$\mathcal{M}_{5}^{\text {Rcstr }}$ & 0.019 & 0.017 & 0.016 & 0.014 & 0.012 & 0.010 \\
\hline \hline$\mu_{\text {hard }}$ & 1.4 & 1.6 & 1.8 & 2.0 & 2.2 & 2.5 \\
\hline $\mathcal{M}_{5}^{\text {Poles }}$ & 0.040 & 0.051 & 0.059 & 0.065 & 0.070 & 0.076 \\
$\mathcal{M}_{5}^{\text {Rcstr }}$ & 0.013 & 0.014 & 0.015 & 0.016 & 0.017 & 0.017 \\
\hline
\end{tabular}

Table 20: $\mathcal{M}_{5}^{\text {Poles }}$ and $\mathcal{M}_{5}^{\text {Rcstr }}$ for different $\mu_{\text {soft }}$ with $\mu_{\text {fac }}=1.45 \mathrm{GeV}$ and $\mu_{\text {hard }}=1.75 \mathrm{GeV}$, for different $\mu_{\text {fac }}$ with $\mu_{\text {soft }}=1.1 \mathrm{GeV}$ and $\mu_{\text {hard }}=1.75$ $\mathrm{GeV}$ and for different $\mu_{\text {hard }}$ with $\mu_{\text {soft }}=1.1 \mathrm{GeV}$ and $\mu_{\text {fac }}=1.45 \mathrm{GeV}$.

\begin{tabular}{|c|c|c|c|c|c|c|c|}
\hline$\mu_{\text {soft }}$ & & 1.0 & 1.05 & 1.1 & 1.15 & 1.2 & 1.25 \\
\hline \multirow{3}{*}{$\mathcal{M}_{5}^{\text {Poles }}$} & LO & 0.035 & 0.027 & 0.022 & 0.018 & 0.015 & 0.013 \\
& NLO & 0.064 & 0.050 & 0.040 & 0.033 & 0.028 & 0.024 \\
& NNLO & 0.10 & 0.075 & 0.057 & 0.045 & 0.036 & 0.030 \\
\hline \multirow{3}{*}{$\mathcal{M}_{5}^{\text {Resum }}$} & LO & 0.019 & 0.016 & 0.014 & 0.013 & 0.011 & 0.010 \\
& NLO & 0.011 & 0.010 & 0.0097 & 0.0092 & 0.0086 & 0.0081 \\
& NNLO & 0.0096 & 0.0075 & 0.0061 & 0.0051 & 0.0043 & 0.0037 \\
\hline
\end{tabular}

Table 21: Size of the moments from the poles and the resummed spectral density at LO, NLO and NNLO for different values of $\mu_{\text {soft }}$.

$$
1.4 \mathrm{GeV} \leq \mu_{\text {hard }} \leq 2.5 \mathrm{GeV}: \quad \Delta m_{P S, c}=50 \mathrm{MeV}
$$

Table 20 and 21 show the scale dependence and the behaviour for the different orders. Compared to the pole scheme, the uncertainty on $\alpha_{s}$ is much improved and amounts to $\Delta m_{P S, c}=20 \mathrm{MeV}$ and $\Delta m_{c}\left(m_{c}\right)=10 \mathrm{MeV}$. As for the bottomium we now investigate the significance of the perturbative contribution. The solid line in figure 12 shows the central mass. Then we just change the perturbative spectral density. The dashed line shows the LO result and in the dotted line we neglect the perturbative contribution completely. For low $n$ we loose stability for the mass though the effect is not as pronounced as in the bottom case since for the charmonium the poles play a more dominant part.

The influence of the separation scale on the masses is shown in table 22. 


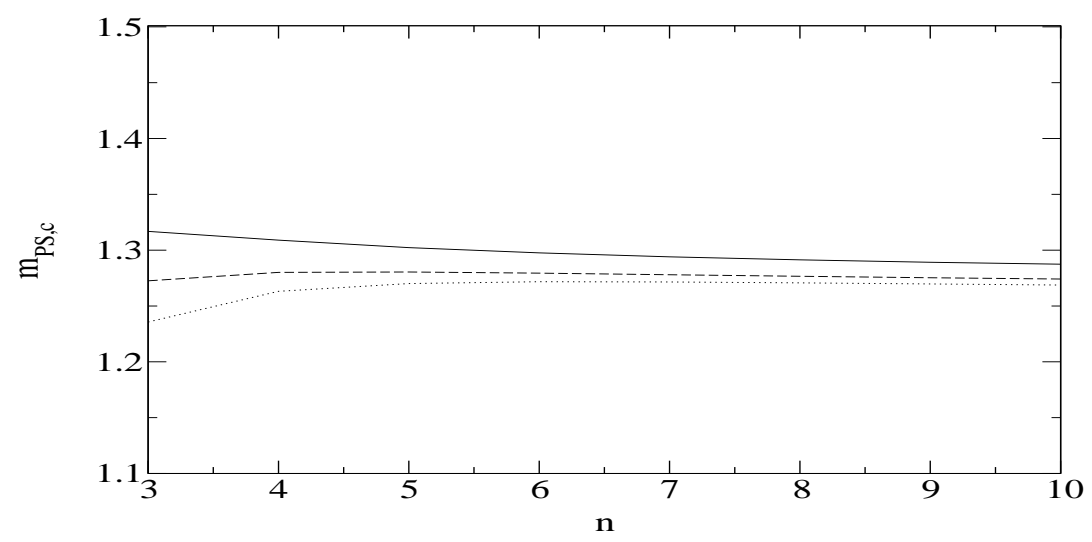

Figure 12: Solid line: central PS-mass; dashed line: perturbative contribution only at LO; dotted line: without perturbative contribution.

\begin{tabular}{|c|c|c|c|c|c|}
\hline$\mu_{\text {sep }}$ & 0.8 & 0.9 & 1.0 & 1.1 & 1.2 \\
\hline$m_{P S, c}$ & 1.353 & 1.326 & 1.300 & 1.277 & 1.255 \\
$m_{c}\left(m_{c}\right)$ & 1.181 & 1.184 & 1.188 & 1.192 & 1.196 \\
\hline
\end{tabular}

Table 22: Change of the masses for different values of $\mu_{\text {sep }}$.

The definition of the PS-mass depends directly on $\mu_{\text {sep }}$, but the $\overline{\mathrm{MS}}$-mass remains very stable and changes only by $\Delta m_{c}\left(m_{c}\right)=8 \mathrm{MeV}$.

Now we turn our attention to the choice of $\xi$. In table 23 the $\overline{\text { MS-mass }}$ is depicted for different $\xi$. As we have done in table 13 we add to the pole contributions the difference from the NNLO-NLO and from NNLO-LO. The change in the $\overline{\mathrm{MS}}$-mass is shown in the third and fourth column. Since the poles are relatively dominant, in principle we would like to use a higher value of $\xi$. But from table 23 one can see that the better expansion is almost compensated by the decreasing sensitivity for $\xi \gtrsim 1$. In addition, the errors from the other input parameters grow. Like in the bottom case, the error from the scales improves for lower $\xi$. The error from the scales is still larger than the estimated uncertainty from the expansion, but in order to be conservative we will use the larger error from the scales for our error estimate. The variation of $\xi$ changes the central value for the mass as well and for $0 \leq \xi \leq 1$ we obtain $\Delta m_{P S, c}=20 \mathrm{MeV}$ which shows a much better behaviour than in the pole scheme. A summary of all contributions to the 


\begin{tabular}{|c|c|c|c|}
\hline$\xi$ & $m_{c}\left(m_{c}\right) /[\mathrm{GeV}]$ & $\Delta m_{c}^{(a)} /[\mathrm{MeV}]$ & $\Delta m_{c}^{(b)} /[\mathrm{MeV}]$ \\
\hline-0.5 & 1.161 & 44 & 73 \\
0 & 1.174 & 35 & 64 \\
0.5 & 1.188 & 31 & 59 \\
1.0 & 1.205 & 30 & 56 \\
1.5 & 1.223 & 29 & 54 \\
2.0 & 1.241 & 30 & 54 \\
3.0 & 1.273 & 31 & 54 \\
4.0 & 1.300 & 34 & 56 \\
\hline
\end{tabular}

Table 23: Change of the $\overline{\mathrm{MS}}$-mass, when adding the difference from NNLONLO to the poles, $\Delta m_{c}^{(a)}$, and the difference from NNLO-LO, $\Delta m_{c}^{(b)}$.

\begin{tabular}{|l|r|r|}
\hline \multicolumn{1}{|c|}{ Source } & $\Delta m_{P S, c}$ & $\Delta m_{c}\left(m_{c}\right)$ \\
\hline Variation of $\mu_{\text {soft }}$ & $85 \mathrm{MeV}$ & $75 \mathrm{MeV}$ \\
Variation of $\mu_{\text {fac }}$ & $65 \mathrm{MeV}$ & $55 \mathrm{MeV}$ \\
Variation of $\mu_{\text {hard }}$ & $50 \mathrm{MeV}$ & $40 \mathrm{MeV}$ \\
Variation of $\mu_{\text {sep }}$ & - & $10 \mathrm{MeV}$ \\
Experimental cross section & $5 \mathrm{MeV}$ & $5 \mathrm{MeV}$ \\
Experimental widths & $20 \mathrm{MeV}$ & $20 \mathrm{MeV}$ \\
Variation of $v_{\text {sep }}$ & $10 \mathrm{MeV}$ & $10 \mathrm{MeV}$ \\
Variation of $\Lambda_{Q C D}$ & $20 \mathrm{MeV}$ & $10 \mathrm{MeV}$ \\
Variation of $\xi$ & $20 \mathrm{MeV}$ & $15 \mathrm{MeV}$ \\
\hline Total error & $124 \mathrm{MeV}$ & $106 \mathrm{MeV}$ \\
\hline
\end{tabular}

Table 24: Single contributions to the error of $m_{P S, c}$ and $m_{c}\left(m_{c}\right)$. 
error is presented in table 24. Finally we obtain the masses:

$$
\begin{aligned}
m_{P S, c}\left(\mu_{\text {sep }}=1.0\right) & =1.300 \pm 0.124 \mathrm{GeV}, \\
m_{c}\left(m_{c}\right) & =1.188 \pm 0.106 \mathrm{GeV} .
\end{aligned}
$$

This value is $59 \mathrm{MeV}$ lower than the central value from the pole scheme. This is no surprise since in the pole scheme the theoretical contributions have large perturbative corrections and the relation between the masses contains large uncertainties as well.

In our previous work on the charm quark mass [17] we obtained a slightly higher charm quark mass of $m_{c}\left(m_{c}\right)=1.23 \pm 0.09 \mathrm{GeV}$. In this work we have chosen an evaluation point of $\xi=0.5$. In the theoretical QCD calculation of the moments in the PS-scheme we have now set the start of the continuum in eq. (31) according to the pole mass which is the appropriate mass definition for free quark production. This leads to a reduction of $m_{c}$ by $25 \mathrm{MeV}$. On the phenomenological side we have included the BES data. This gives a better control in the region between 3.8 and $4.6 \mathrm{GeV}$ where the assumptions of quark-hadron-duality cannot be expected to work well. Finally we have extended the error analysis.

\section{Comparison to other mass determinations}

Now we compare our value for the charm quark mass to other determinations. In this and the next section the $\overline{\mathrm{MS}}$-masses are always evaluated at their own scale, $m_{c}=m_{c}\left(m_{c}\right)$ and $m_{b}=m_{b}\left(m_{b}\right)$. The basis of the charmonium sum rules was already laid in 47, 48. Since then, many researchers have extracted the charm quark mass from the sum rules. In [9] the pole mass was determined from perturbation theory to NLO resulting in a value of $M_{c}=1.46 \pm 0.07 \mathrm{GeV}$. In a second investigation [10,49] the analysis has been performed in the $\overline{\mathrm{MS}}$-scheme with perturbation theory to NLO. Using the NLO relation to the pole mass the author obtains $m_{c}=1.26 \pm 0.05 \mathrm{GeV}$ and $M_{c}=1.42 \pm 0.03 \mathrm{GeV}$. The author has also performed an analysis using resummation in $\mathrm{LO}$ with a value of $M_{c}=1.45 \pm 0.07 \mathrm{GeV}$. In our analysis the increased value of the pole mass is essentially due to large Coulomb contributions which have not been included in former analyses. As a consequence, the error becomes larger as well.

The charm quark mass can also be derived from direct application of NRQCD to hadronic bound states. The authors of [50, 51] have studied the energy level of the charmonium ground state. They conclude a $\overline{\mathrm{MS}}$ mass of $m_{c}=1.241 \pm 0.015 \mathrm{GeV}$ where the error is from the variation of $\alpha_{s}$ only. In [15] a similar analysis was performed for the pole mass with 
the result $M_{c}=1.88_{-0.13}^{+0.22} \mathrm{GeV}$. In 52] NRQCD was applied to the mass difference between the $B$ and $D$ mesons with $m_{c}=1.21 \pm 0.11 \mathrm{GeV}$. Further improvement of these determinations may be possible in the near future. However, these determinations face the problem that the contributions from NRQCD must be directly evaluated at low energies close to threshold whereas in the sum rules the theoretical expansions can be evaluated in a perturbative region. Furthermore, nonperturbative effects may have a significant impact on the charmonium energy levels.

During the last years several lattice analyses have been performed for the charm quark mass [53, 54, 55, 56, 57, 58, with rather widespread results. The most recent one [58] obtains $m_{c}=1.26 \pm 0.04 \pm 0.12 \mathrm{GeV}$. This calculation was done at a lattice spacing of $a \approx 0.07 \mathrm{fm}$. Though it was done in quenched QCD, the authors expect a minor decrease of $5 \%$ for the unquenched case. Here also a discussion on the previous lattice analyses can be found. Very recently, two preliminary results [59, 60] from different lattice groups have been presented with $m_{c}=1.314 \pm 0.05 \mathrm{GeV}$ and $m_{c}=1.27 \pm 0.05 \mathrm{GeV}$ (statistical error only) respectively.

Ref. 61] has applied pseudoscalar sum rules to heavy-light quark systems. Values of $m_{c}=1.10 \pm 0.04 \mathrm{GeV}$ and $m_{b}=4.05 \pm 0.06 \mathrm{GeV}$ were deduced. A discussion on this work can be found in 62.

In some recent works the charm quark mass has been determined from charmonium sum rules on perturbative grounds without Coulomb resummation. The authors of 63 have used moment sum rules for the charmonium. They use moments of $n \approx 1-4$ and argue that resummation is not necessary for such low values of $n$. On the theoretical side $\Pi(s)$ is calculated up to $O\left(\alpha_{s}^{2}\right)$ at a scale of $\mu=3 \mathrm{GeV}$. This is compared to the phenomenological part which includes the $J / \psi$ and $\psi^{\prime}$ resonances and the data from BES [38] above the $D$ threshold. They obtain $m_{c}=1.30 \pm 0.03 \mathrm{GeV}$. A similar analysis for the bottom yields $m_{b}=4.21 \pm 0.05 \mathrm{GeV}$. In 64] a contour integration was performed to apply the Cauchy sum rules. The integral was closed at an energy of $5 \mathrm{GeV}$. At this scale the high energy approximation was used to calculate $\Pi(s)$ up to $O\left(\alpha_{s}^{2}\right)$. As in [63], this was compared to the two lowest $\psi$-resonances plus the continuum data [38]. The result is $m_{c}=1.37 \pm 0.09$ $\mathrm{GeV}$. Very recently, the authors of [65] obtain $m_{c}=1.289_{-0.045}^{+0.040} \mathrm{GeV}$ from a comparison of the perturbative spectral density to continuum data. Ref. 66] has presented an update of the SVZ sum rules [2] with perturbation theory at NNLO. They extract the gluon condensate and a charm quark mass of $m_{c}=1.275 \pm 0.015 \mathrm{GeV}$.

However, in light of the present work it seems doubtful that a reliable determination of the charm quark mass from the charmonium system can be achieved by a pure perturbative evaluation without resummation as in 
63, 64, 65, 66. To clarify this point, let us return to the numerical analysis. Choosing $\xi$ sufficiently large and $n$ small enough, one can easily approach a region where the perturbative contribution represents the dominant part. One could then expect that the use of perturbation theory and its relative small scale dependence will give a reasonable approximation. Nevertheless, this conclusion is misleading.

For values of $\xi=4$ and $n=4$ the moment from the perturbative contribution exceeds the poles by $30 \%$ (for $n \leq 3$ the phenomenological continuum is very dominant and the analysis becomes unstable). Nevertheless, a variation of the soft scale gives an error of $\Delta m_{c}=110 \mathrm{MeV}$ whereas the error from the hard scale is $\Delta m_{c}=70 \mathrm{MeV}$ and this error could even be improved by using a high energy approximation. For even higher $\xi$ the result is similar: for $\xi=6$ and $n=4$ the perturbative contribution is twice as large as the pole contributions, but the error from $\mu_{\text {soft }}$ gives $\Delta m_{c}=125 \mathrm{MeV}$ and from the error from $\mu_{\text {hard }}$ amounts to $\Delta m_{c}=90 \mathrm{MeV}$. Though the pole contributions are relatively suppressed, the mass reacts much stronger on the remaining uncertainties. This behaviour can already be seen in table 23. Ever higher $\xi$ do not improve the accuracy any more but in same way as the theoretical expansion improves the sensitivity on the mass is lost. Indeed, if we set the pole contribution to zero in our analysis, $m_{c}$ would drop by approximately $300 \mathrm{MeV}$ even for very high $\xi$ and the analysis could not be trusted any more.

That a description without inclusion of the theoretical poles is insufficient can already be seen from the quantum mechanical sum rules for the mass (25). On the phenomenological side, the main dependence on the mass originates already from the first bound states, even if the continuum part dominates the moments. The contributions from the poles, starting from $O\left(\alpha_{s}^{3}\right)$, must be included in the theoretical description as well to obtain a reliable mass determination. As was discussed in [66], the charmonium system is not well described as a Coulomb system. In particular, the expansion for the higher states cannot be trusted and the effective potential may differ from the Coulombic one. However, it is indispensable to use the terms from resummation for a determination of the mass. The most important contribution to the sum rules originates already from the ground state (2425). The quantum mechanical sum rules show clearly that fixed-order perturbation theory in a system whose ground state is governed by a Coulomb-similar potential leads to an instable and unreliable sum rule for the mass.

However, it should be kept in mind that a Coulomb-dominated description of the charmonium system stands on less firm grounds than for the upsilon system whose energy is sufficiently large to allow for a reliable resummation. This is also reflected in the fact that the relative error of $m_{c}$ is almost a factor 4 larger than the relative error of $m_{b}$. 


\begin{tabular}{|l|l|l|}
\hline \multicolumn{3}{|c|}{ QCD sum rules } \\
\hline Authors & $M_{b} /[\mathrm{GeV}]$ & $m_{b} /[\mathrm{GeV}]$ \\
\hline V [22] & $4.83 \pm 0.01$ & \\
KPP [67] & $4.75 \pm 0.04$ & $4.20 \pm 0.10$ \\
MY [68] & $4.80 \pm 0.06$ & $4.21 \pm 0.11$ \\
PP [13] & $4.84 \pm 0.08$ & $4.19 \pm 0.06$ \\
JP [27, 69] & $4.25 \pm 0.08$ \\
BS [70] & $4.17 \pm 0.05$ \\
H [14,71, 72] & $4.98 \pm 0.125$ & $4.24 \pm 0.10$ \\
This work & NRQCD potential \\
\hline \multicolumn{3}{|c|}{ Lattice QCD } \\
\hline PY [15] & $5.015_{-0.07}^{+0.11}$ & $4.45_{-0.03}^{+0.05}$ \\
BSV [50, 51] & $4.19 \pm 0.03$ \\
P [52] & $4.21 \pm 0.09$ \\
\hline \multicolumn{3}{|c|}{} \\
\hline A et al. 73] & $4.35 \pm 0.23$ \\
GGMR [7] & $4.26 \pm 0.09$ \\
\hline
\end{tabular}

Table 25: Some references to the bottom quark mass.

During the last years much effort has been dedicated to the determination of the bottom quark mass. The methods which have been employed were mainly based on QCD sum rules for the upsilon system, NRQCD for the bound states or lattice QCD. We have listed some of these results in table 25. A more complete list of references can be found in [1]. We can directly compare our work to previous sum rule analyses. As detailed discussions about the advances and drawbacks of these analyses can be found in [14,70,75], here we rather want to point out some interesting differences. For the comparison we will use the work by Hoang [14,71,72 where the most extensive analysis has been presented. In that moment sum rules the theoretical moments were directly expanded for small energies around threshold. With help of a contour integration this could be used to calculate the moments via the inverse Laplace transform. Since the approach has focused on the nonrelativistic properties of the upsilon system, for a comparison we now set the perturbative contributions in our analysis to zero. For a closer comparison we choose a value of $\xi=0$ where the analysis of Hoang was performed. Two effects become important: The central value of the mass decreases by $25 \mathrm{MeV}$. Second, at $\xi=0$ the poles are more dominant and the influence of the perturbative contributions is reduced. For $\xi=0$, without perturbation theory and for $7 \leq n \leq 10$ we obtain $m_{b}=4.17 \mathrm{GeV}$. For $n \lesssim 6$ the analysis becomes 
unstable. Three differences remain. For our central mass we have used a factorisation scale of $\mu_{f a c}=3.5 \mathrm{GeV}$ whereas Hoang has performed a scan over $2.5 \mathrm{GeV} \leq \mu_{f a c} \leq 10.0 \mathrm{GeV}$ which roughly corresponds to a central value of $\mu_{f a c}=5.0 \mathrm{GeV}$. Second, for the parametrisation of the phenomenological continuum Hoang has used a continuum threshold of $\sqrt{s_{0}}=10.56 \mathrm{GeV}$ which corresponds to the start of $B \bar{B}$ production. As discussed in chapter 6 , we have chosen a value of $\sqrt{s_{0}}=11.0 \mathrm{GeV}$ to parametrise the non-resonant part of the spectral density. Using $\mu_{f a c}=5.0 \mathrm{GeV}$ and $\sqrt{s_{0}}=10.56 \mathrm{GeV}$ we finally arrive at $m_{b}=4.14 \mathrm{GeV}$. In his work, Hoang has estimated the effect of a finite charm quark mass to be $\Delta m_{b} \approx-30 \mathrm{MeV}$. For a massless charm quark he obtains $m_{b}=4.20 \mathrm{GeV}$ which is $60 \mathrm{MeV}$ higher than our result for similar input parameters. Our error is larger than his one by a factor of two. Hoang has used a $\chi^{2}$-fit with several moments. In this way he gets a cancellation between theoretical contributions of the $\chi^{2}$-function. In our analysis we keep the mass as a function of $n$ which serves as additional check for the stability of the sum rules. Furthermore, at $\xi=0.5$ the mass reacts stronger on a variation of the parameters. But as was discussed in the numerical analysis, in this way the control over the analysis is improved and we believe that we have thus reduced the systematic uncertainties of the method.

Now we want to comment on a recent work on massless contributions to the heavy quark correlator [76, 77]. Here it was shown that at $O\left(\alpha_{s}^{3}\right)$ the correlator contains a three-gluon massless intermediate state. Its contribution to the moments $\mathcal{M}_{n}(\xi=0)$ contains a divergent term for $s=0$ and $n \geq 4$. Thus the authors have concluded that the moment sum rules can only be reliably evaluated at $n<4$. We believe that this claim is unfounded as was already noticed in 48. First, up to now the sum rule analyses contain perturbative contributions up to $O\left(\alpha_{s}^{2}\right)$ and higher order terms from resummation. At this order the 3-gluon cut was not and should not be included in the analysis and all quantities are well defined. Furthermore, even if the calculation was including the full $O\left(\alpha_{s}^{3}\right)$ contributions, these terms must not be taken into account. The terms from the three gluon cut mainly correspond to light quark production and the divergent parts at $s=0$ are entirely due to light quark production as the heavy quark pair only gives a contribution above $s>4 M^{2}$. Since they are not included in the phenomenological part they also should not appear in the theoretical part and must be explicitly subtracted from the perturbative contributions. The remaining ambiguity which results from the difficulty to separate the light and heavy quark production in the dispersion integral above $s>4 M^{2}$ is a finite effect of $O\left(\alpha_{s}^{3}\right)$ and can be completely neglected within the uncertainty of this work.

As discussed in 32, the problem is of more general nature. Already 
starting at $O\left(\alpha_{s}^{2}\right)$, it is no longer true that a specific flavour current $j_{\mu}=$ $\bar{Q} \gamma_{\mu} Q$ contains only heavy quark production neither that all heavy quark production originates from this current. As was shown in chapter 4 for the diagrams of fig. 3, also light-light and heavy-light correlators contain heavy quark production. Since only the total electromagnetic current, including a sum over all flavours, is a physical observable, the single flavour production is not uniquely defined. The crucial point is to set up two identical quantities: the phenomenological and the theoretical side should be defined is such a way that they contain the same contributions. The higher the order this may be a more and more complicated task.

\section{Conclusions}

In this work we have obtained the following values for the charm and bottom quark masses:

$$
\begin{aligned}
& M_{c}=1.75 \pm 0.15 \mathrm{GeV}, \quad m_{c}=1.19 \pm 0.11 \mathrm{GeV} \\
& M_{b}=4.98 \pm 0.125 \mathrm{GeV}, \quad m_{b}=4.24 \pm 0.10 \mathrm{GeV} .
\end{aligned}
$$

As in the last section we evaluate the $\overline{\mathrm{MS}}$-masses at their own scale, $m_{c, b}=$ $m_{c, b}\left(m_{c, b}\right)$. Now we summarise the key features of this analysis.

In section 2 we have presented a rather complete setup for the quantum mechanical sum rules in the Coulomb potential. The correlator contains poles below and a continuum above threshold. The poles only start with a power of $O\left(\alpha^{3}\right)$, but exhibit an exponential behaviour (16) in the Borel sum rules or a sensitive power behaviour in the moment sum rules (24). Therefore the relative size of the poles depends strongly on either the Borel parameter in the Borel sum rules or on $n$ and $\xi$ in the moment sum rules. The analysis must be performed in a certain sum rule window for $\tau$ or for $n$ and $\xi$ respectively to guarantee a reliable theoretical calculation and sensitivity to the phenomenological parameters.

In the field theory case the expansion of the Green's function is known up to NNLO in the framework of NRQCD. The Green's function is directly evaluated at $s_{0}=-4 m^{2} \xi$ and in this way we avoid to sum up the energy levels individually where the expansion is badly convergent. The spectral density can be obtained from the imaginary part of the Green's function and the pole contributions from the difference between the full and the continuum result. The resulting moments depend on three scales: $\mu_{\text {soft }}, \mu_{\text {fac }}$ and $\mu_{\text {hard }}$. In particular the dependence on $\mu_{\text {soft }}$ is relatively strong and presents the dominant contribution to the error. Since the pole mass contains renormalon ambiguities we have also performed the analysis for the PS-mass which can be 
perturbatively related to the $\overline{\mathrm{MS}}$-mass. Whereas the PS-mass by definition depends on the separation scale which was used to subtract the long-distance potential, this dependence cancels in the transition to the $\overline{\mathrm{MS}}$-mass to a large extend. We have then included the perturbative contributions up to $O\left(\alpha_{s}^{2}\right)$. They are necessary to construct the spectral density above threshold for the full energy range and to guarantee the stability of the mass in a region of small $n$.

One of the great virtues of the method of QCD sum rules is the analytic dependence on the theoretical and phenomenological parameters. Thus we have investigated their importance and influence on the analysis. For the determination of the masses we have used central values for all parameters. These values were not motivated by any optimisation or stability requirement, but only grounded on general considerations. Each was varied in a suitably large chosen window for the error estimate. Only the lower value of $\mu_{\text {soft }}$ was also limited by the convergence of the nonrelativistic expansion. Finally, all errors have been added quadratically.

We have set up the sum rules for an arbitrary evaluation point $\xi$. With this parameter it is possible to shift the moments into a more perturbative region for higher $\xi$ or into a region more sensitive to the bound state energies and the mass for lower $\xi$. We have used $\xi=0.5$ both for the charmonium and bottomium. In fact, moving away from the threshold region and loosing sensitivity on the mass, the scale dependence is even a bit larger than at $\xi=0$. But the convergence of the theoretical expansions is improved and the theoretical contributions more equally distributed among the different terms, in particular, the pole contributions do not play such a dominant role. Thus we believe that we have reduced the systematic uncertainties in this sum rules which cannot be accounted for by a variations of the scales.

We would like to emphasise a remarkable property of this analysis: Once a particular set of (central) values for $\mu_{\text {soft }}, \mu_{f a c}$ and $\mu_{\text {hard }}$ is chosen, the $\overline{\mathrm{MS}}$ masses remain very stable over a large range of values for $n, \xi$ or $\mu_{\text {sep }}$. In gen-

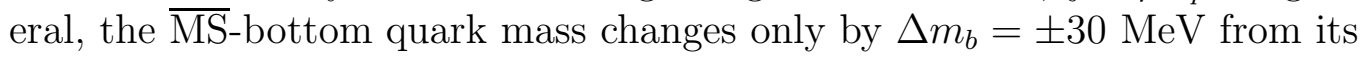
central value for $4 \leq n \leq 15$ and the charm quark mass by $\Delta m_{c}= \pm 15 \mathrm{MeV}$ for $3 \leq n \leq 15$. Varying $\xi$ between $-0.25 \leq \xi \leq 2$ the bottom quark mass changes by $\Delta m_{b}={ }_{-40}^{+50} \mathrm{MeV}$ and the charm quark mass by $\Delta m_{c}={ }_{-30}^{+50} \mathrm{MeV}$ for $-0.5 \leq \xi \leq 2$. The variation of $\mu_{\text {sep }}$ changes the PS-mass significantly since its definition depends on $\mu_{\text {sep }}$. But in the relation to the $\overline{\mathrm{MS}}$-mass this change is almost completely cancelled and the bottom quark mass changes by $\Delta m_{b}= \pm 7 \mathrm{MeV}$ for $1.0 \mathrm{GeV} \leq \mu_{\text {sep }} \leq 3.0 \mathrm{GeV}$ and the charm quark mass by $\Delta m_{c}= \pm 8 \mathrm{MeV}$ for $0.8 \mathrm{GeV} \leq \mu_{\text {sep }} \leq 1.2 \mathrm{GeV}$. These results are astonishing since the variation of these parameters corresponds to largely different relative influence among the theoretical contributions. Thus we hope 
that we were able to set up a consistent framework in which the physics of the relevant energy region, apart from the remaining uncertainties in the nonrelativistic and perturbative expansions, has been correctly described.

Let us finally summarise the achieved status. In our analysis, several contributions seem to be under good control: The perturbative expansion, as it has been incorporated in our analysis, converges reasonably well. The condensates only give a negligible contribution to the upsilon or charmonium. On the phenomenological side, for the upsilon the first six resonances have been measured. For the non-resonant continuum part, quark-hadron duality has been used. In the charmonium system, the experimental situation has improved recently. Besides the first six resonances also the cross section between $2.0 \mathrm{GeV}$ and $4.8 \mathrm{GeV}$ has been measured. Above this energy we again use quark-hadron duality. Nevertheless, the most important contribution is already given by the first two poles.

Of decisive importance for the determination of the masses is the threshold behaviour. The method of QCD sum rules is a very powerful tool to extract the masses since - by the choice of $n$ and $\xi$ - it can react very sensitive to the threshold. Thus, large theoretical uncertainties only lead to a relatively small shift in the masses. The main uncertainties indeed come from the threshold expansion of NRQCD. The largest potential for an improvement of the analysis lies in a further understanding of this energy region. In particular, the knowledge of the Green's function at NNNLO could help to reduce the error.

The method of QCD sum rules is based on the assumptions of quarkhadron duality. With the development of NRQCD is has become clear that the pole contributions must be included in the theoretical description for a correct comparison between the theoretical and phenomenological part. The theoretical description is based on the operator product expansion and can be performed in a perturbative region where all expansions converge well. However, this cannot be used for an ever increasing precise determination of the mass. Since on the phenomenological side the dependence on the mass originates mainly from the first bound states, the sensitivity on the mass decreases in a similar way as the theoretical expansions improve. In our analysis we have tried to balance these contributions by an appropriate choice of $\xi$ and $n$. Without significant progress in the theoretical description it seems that further substantial improvement will be difficult to achieve.

\section{Acknowledgements}

It is a pleasure to thank Matthias Jamin for numerous discussions, collaboration in parts of this work and reading the manuscript. I would like to thank Nora Brambilla, Antonio Pich, Jorge Portolés, Pedro Ruiz and Anto- 
nio Vairo for helpful and interesting discussions and Antonio Pich for reading the manuscript. This work has been supported in part by TMR, EC contract No. ERB FMRX-CT98-0169, by MCYT (Spain) under grant FPA2001-3031, and by ERDF funds from the European Commission. I thank the Deutsche Forschungsgemeinschaft for financial support.

\section{A Potential}

In NRQCD the Green's function obeys the Schrödinger equation:

$$
\begin{aligned}
(- & \frac{\Delta_{x}}{M}-\frac{\Delta_{x}^{2}}{4 M^{3}}+V_{C}(x)+\frac{\alpha_{s}}{4 \pi} \Delta_{1} V(x)+\frac{\alpha_{s}^{2}}{16 \pi^{2}} \Delta_{2} V(x) \\
& \left.+\Delta_{N A} V(x)+\Delta_{B F} V(x)+\frac{k^{2}}{M}\right) G(\mathbf{x}, \mathbf{y}, k)=\delta^{(3)}(\mathbf{x}-\mathbf{y}) .
\end{aligned}
$$

$V_{C}(x)$ is the Coulomb potential, $\Delta_{N A} V(x)$ the nonabelian part of the quarkantiquark potential, $\Delta_{B F} V(x)$ the Breit-Fermi potential and the terms $\Delta_{1} V(x)$ and $\Delta_{2} V(x)$ contain the first and second order perturbative correction to the Coulomb potential. The explicit expressions read:

$$
\begin{aligned}
V_{C}(x)= & -\frac{C_{F} \alpha_{s}}{x}, \quad x=|\mathbf{x}| \\
\Delta_{N A} V(x)= & -C_{A} C_{F} \frac{\alpha_{s}^{2}}{2 M x^{2}}, \\
\Delta_{B F} V(x)= & \frac{C_{F} \alpha_{s} \pi}{M^{2}} \delta^{(3)}(\mathbf{x})-\frac{C_{F} \alpha_{s}}{2 M^{2} x}\left(\mathbf{p}^{2}+\frac{1}{x^{2}} \mathbf{x}(\mathbf{x p}) \mathbf{p}\right)+\frac{3 C_{F} \alpha_{s}}{2 M^{2} x^{3}} \mathbf{S L} \\
& -\frac{C_{F} \alpha_{s}}{2 M^{2}}\left(\frac{\mathbf{S}^{2}}{x^{3}}-\frac{3(\mathbf{S x})^{2}}{x^{5}}-\frac{4 \pi}{3}\left(2 \mathbf{S}^{2}-3\right) \delta^{(3)}(\mathbf{x})\right) \\
\Delta_{1} V(x)= & V_{C}(x)\left(a_{1}+2 b_{0} \gamma_{E}+2 b_{0} \ln (x \mu)\right), \\
\Delta_{2} V(x)= & V_{C}(x)\left(a_{2}+b_{0}^{2}\left(\pi^{2} / 3+4 \gamma_{E}^{2}\right)+2 \gamma_{E}\left(b_{1}+2 b_{0} a_{1}\right)\right. \\
& \left.+\left(2 b_{1}+4 b_{0} a_{1}+8 b_{0}^{2} \gamma_{E}\right) \ln (x \mu)+4 b_{0}^{2} \ln ^{2}(x \mu)\right)
\end{aligned}
$$

with the constants

$$
\begin{aligned}
b_{0} & =11-\frac{2 n_{f}}{3} \\
b_{1} & =\frac{34}{3} C_{A}^{2}-\frac{20}{3} C_{A} T n_{f}-4 C_{F} T n_{f}=102-12.67 n_{f}, \\
a_{1} & =\frac{31}{9} C_{A}-\frac{20}{9} T n_{f}=10.33-1.11 n_{f}
\end{aligned}
$$




$$
\begin{aligned}
a_{2}= & \left(\frac{4343}{162}+4 \pi^{2}-\frac{\pi^{4}}{4}+\frac{22}{3} \zeta(3)\right) C_{A}^{2}-\left(\frac{1798}{81}+\frac{56}{3} \zeta(3)\right) C_{A} T n_{f} \\
& -\left(\frac{55}{3}-16 \zeta(3)\right) C_{F} T n_{f}+\left(\frac{20}{9} T n_{f}\right)^{2} \\
= & 456.75-66.35 n_{f}+1.23 n_{f}^{2},
\end{aligned}
$$

where $C_{F}=4 / 3, C_{A}=3$ and $T=1 / 2$. The coefficient $a_{2}$ was first calculated in [78] and later corrected to the above given value [79].

\section{B Potential-Subtracted mass}

The PS-mass is defined by [19]

$$
\begin{aligned}
\delta m\left(\mu_{\text {sep }}\right) & =-\frac{1}{2} \int_{|\mathbf{q}|<\mu_{\text {sep }}} \frac{d^{3} q}{(2 \pi)^{3}} V(q), \\
m_{P S}\left(\mu_{\text {sep }}\right) & =M-\delta m\left(\mu_{\text {sep }}\right) .
\end{aligned}
$$

The subtracted potential $V\left(r, \mu_{\text {sep }}\right)$ is related to the potential in momentum space $V(q)$ and $\delta m\left(\mu_{\text {sep }}\right)$ :

$$
\begin{aligned}
V(q) & =-\frac{4 \pi C_{F} \alpha_{s}(\mathbf{q})}{\mathbf{q}^{2}}\left(1+a_{1} \frac{\alpha_{s}(\mathbf{q})}{4 \pi}+a_{2}\left(\frac{\alpha_{s}(\mathbf{q})}{4 \pi}\right)^{2}\right), \\
V(r) & =\int \frac{d^{3} q}{(2 \pi)^{3}} e^{i \mathbf{q r}} V(q), \\
V\left(r, \mu_{\text {sep }}\right) & =V(r)+2 \delta m\left(\mu_{\text {sep }}\right),
\end{aligned}
$$

with $a_{1}$ and $a_{2}$ as in eq. (58). Performing the Fourier transformation one obtains the relation between PS- and pole mass:

$$
\begin{aligned}
m_{P S}\left(\mu_{\text {sep }}\right) & =M\left[1+a(\mu) r_{P S}^{(1)}\left(\mu_{\text {sep }}\right)+a^{2}(\mu) r_{P S}^{(2)}\left(\mu, \mu_{\text {sep }}\right)+a^{3}(\mu) r_{P S}^{(3)}\left(\mu, \mu_{\text {sep }}\right)\right], \\
r_{P S}^{(1)}\left(\mu_{\text {sep }}\right) & =-C_{F} \frac{\mu_{\text {sep }}}{M}, \quad r_{P S}^{(2)}\left(\mu, \mu_{\text {sep }}\right)=-C_{F} \frac{\mu_{\text {sep }}}{M} \frac{w_{1}\left(\mu, \mu_{\text {sep }}\right)}{4}, \\
r_{P S}^{(3)}\left(\mu, \mu_{\text {sep }}\right) & =-C_{F} \frac{\mu_{\text {sep }}}{M} \frac{w_{2}\left(\mu, \mu_{\text {sep }}\right)}{16},
\end{aligned}
$$

with $a=\alpha_{s} / \pi$ and the functions

$$
\begin{aligned}
& w_{1}\left(\mu, \mu_{\text {sep }}\right)=a_{1}-b_{0}\left(\ln \frac{\mu_{\text {sep }}^{2}}{\mu^{2}}-2\right), \\
& w_{2}\left(\mu, \mu_{\text {sep }}\right)=a_{2}-\left(2 a_{1} b_{0}+b_{1}\right)\left(\ln \frac{\mu_{\text {sep }}^{2}}{\mu^{2}}-2\right)+b_{0}^{2}\left(\ln ^{2} \frac{\mu_{\text {sep }}^{2}}{\mu^{2}}-4 \ln \frac{\mu_{\text {sep }}^{2}}{\mu^{2}}+8\right),
\end{aligned}
$$


with $b_{0}$ and $b_{1}$ from eq. (58). Using the three-loop result between pole and $\overline{\mathrm{MS}}$-mass one can relate the PS- and $\overline{\mathrm{MS}}$-mass:

$$
\begin{aligned}
m_{P S}\left(\mu_{\text {sep }}\right)= & m\left[1+a(m)\left(k_{1}-C_{F} \frac{\mu_{\text {sep }}}{m}\right)+a^{2}(m)\left(k_{2}-C_{F} \frac{\mu_{\text {sep }}}{m} \frac{w_{1}\left(m, \mu_{\text {sep }}\right)}{4}\right)\right. \\
& \left.+a^{3}(m)\left(k_{3}-C_{F} \frac{\mu_{\text {sep }}}{m} \frac{w_{2}\left(m, \mu_{\text {sep }}\right)}{16}\right)\right], \\
k_{1}= & C_{F}, \quad k_{2}=13.443-1.041 n_{f}, \\
k_{3}= & 190.595-26.655 n_{f}+0.653 n_{f}^{2},
\end{aligned}
$$

where $m=m_{\overline{\mathrm{MS}}}\left(m_{\overline{\mathrm{MS}}}\right)$ is the $\overline{\mathrm{MS}}$-mass evaluated at its own scale.

\section{References}

[1] D.E. Groom et AL., Eur. Phys. J. C 15 (2000) 1.

[2] M.A. Shifman, A.I. Vainshtein and V.I. Zakharov, Nucl. Phys. B 147 (1979) 385, Nucl. Phys. B 147 (1979) 448.

[3] L.J. Reinders, H. Rubinstein and S. Yazaki, Phys. Rep. 127 (1985) 1.

[4] S. NARISOn, QCD Spectral Sum Rules, World Scientific (1989).

[5] H.J. Rothe, Lattice gauge theories, World Scientific (1992).

[6] I. Montvay And G. Münster, Quantum fields on a lattice, Cambridge University Press (1994).

[7] J. Gasser and H. Leutwyler, Nucl. Phys. B 250 (1985) 465.

[8] A. PICH, Rept. Prog. Phys. 58 (1995) 563.

[9] C.A. Dominguez, G.R. Gluckman and N. Paver, Phys. Lett. B 333 (1994) 184.

[10] S. Narison, Phys. Lett. B 341 (1994) 73.

[11] W.E. Caswell and G.E. Lepage, Phys. Lett. B 167 (1986) 437.

[12] G.T. Bodwin, E. Braaten and G.P. Lepage, Phys. Rev. D 51 (1995) 1125.

[13] A.A. Penin And A.A. Pivovarov, Nucl. Phys. B 549 (1999) 217. 
[14] A.H. Hoang, Phys. Rev. D 59 (1999) 014039.

[15] A. Pineda and F.J. Ynduráin, Phys. Rev. D 58 (1998) 094022.

[16] V.A. Novikov, M.A. Shifman, A.I. Vainshtein and V.I. ZaKharov, Sov. J. Nucl. Phys. 32 (1980) 840, Nucl. Phys. B 191 (1981) 301.

[17] M. Eidemüller And M. Jamin, Phys. Lett. B 498 (2001) 203.

[18] M. Beneke, Phys. Rep. 317 (1999) 1.

[19] M. Beneke, Phys. Lett. B 434 (1998) 115.

[20] J. Bell and R. Bertlmann, Nucl. Phys. B 177 (1981) 218.

[21] P. Pascual and R. Tarrach, QCD: Renormalisation for the Practitioner, Lecture Notes in Physics 194, Springer (1984).

[22] M.B. Voloshin, Int. J. Mod. Phys. A 10 (1995) 2865.

[23] M.J. Strassler And M.E. Peskin, Phys. Rev. D 43 (1991) 1500.

[24] A.H. Hoang and T. Teubner, Phys. Rev. D 58 (1998) 114023.

[25] M. Eidemüller And M. Jamin, Nucl. Phys. 96 (Proc. Suppl.) (2001) 404.

[26] A.H. Hoang, M.C. Smith, T. Stelzer and S. Willenbrock Phys. Rev. D 59 (1999) 114014.

[27] M. Jamin And A. Pich, Nucl. Phys. B 507 (1997) 334.

[28] S.C. Generalis, Dissertation, The Open University, Milton Keynes, England (1984).

[29] D.J. Broadhurst, J. Fleischer und O.V. Tarasov, Z. Physik C 60 (1993) 287.

[30] K.G. Chetyrinin, J.H. Kühn and M. Steinhauser, Nucl. Phys. B 482 (1996) 213.

[31] K.G. Chetyrkin, J.H. Kühn and M. Steinhauser, Nucl. Phys. B 505 (1997) 40.

[32] J. Portolés and P.D. Ruiz-Femenía, Eur. Phys. J. C 24 (2002) 439. 
[33] A.H. Hoang, M. Jezabek, J.H. Kühn and T. Teubner, Phys. Lett. B 338 (1994) 330.

[34] D.J. Broadhurst, P.A. Baikov, V.A. Ilyin, J. Fleischer, O.V. Tarasov and V.A. Smirnov, Phys. Lett. B 329 (1994) 103.

[35] S.N. Nikolaev and A.V. Radyushkin, Nucl. Phys. B 213 (1983) 285.

[36] S.N. Nikolaev and A.V. Radyushkin, Phys. Lett. B 124 (1983) 243.

[37] D.J. Broadhurst and S.C. Generalis, Phys. Lett. B 165 (1985) 175 .

[38] J.Z. Bai et Al. (BeS Collaboration), Phys. Rev. Lett. 88 (2002) 101802.

[39] K.G. Chetyrkin, Phys. Lett. B 391 (1997) 402.

[40] M. Eidemüller, hep-ph/0210247.

[41] K. Melnikov and T. van Ritbergen, Phys. Lett. B 482 (2000) 99.

[42] K.G. Chetyrkin and M. Steinhauser, Nucl. Phys. B 573 (2000) 617.

[43] N. Brambilla, A. Pineda, J. Soto and A. Vairo, Phys. Lett. B 470 (1999) 215.

[44] B.A. Kniehl And A.A. Penin, Nucl. Phys. B 577 (2000) 197.

[45] B.A. Kniehl, A.A. Penin, V.A. Smirnov and M. Steinhauser, Nucl. Phys. B 635 (2002) 357.

[46] A.A. Penin and M. Steinhauser, Phys. Lett. B 538 (2002) 335.

[47] V.A. Novikov, L.B. Okun, M.A. Shifman, A.I. Vainshtein, M.B. Voloshin and V.I. Zakharov, Phys. Rev. Lett. 38 (1977) 626.

[48] V.A. Novikov, L.B. Okun, M.A. Shifman, A.I. Vainshtein, M.B. Voloshin and V.I. Zakharov, Phys. Rep. 41 (1978) 1.

[49] S. Narison, Nucl. Phys. 74 (Proc. Suppl.) (1999) 304. 
[50] N. Brambilla, Y. Sumino and A. Vairo, Phys. Lett. B 513 (2001) 381.

[51] N. Brambilla, Y. Sumino and A. Vairo, Phys. Rev. D 65 (2002) 034001.

[52] A. Pineda, JHEP 0106 (2001) 022.

[53] C.R. Allton, M. Ciuchini, M. Crisafulli, E. Franco, V. LuBicz and G. Martinelli, Nucl. Phys. B 431 (1994) 667.

[54] A. Kronfeld, Nucl. Phys. 63 (Proc. Suppl.) (1998) 311.

[55] V. Giménez, L. Giusti, F. Rapuano and M. Talevi, Nucl. Phys. B 540 (1999) 472.

[56] A. Bochkarev and P. De Forcrand, Nucl. Phys. B 477 (1996) 489.

[57] A. Bochkarev and P. De Forcrand, Nucl. Phys. 53 (Proc. Suppl.) (1997) 305.

[58] D. Bećirević, V. Lubicz and G. Martinelli, Phys. Lett. B 524 (2002) 115.

[59] J. Rolf And S. Sint, Nucl. Phys. 106 (Proc. Suppl.) (2002) 239.

[60] K.J. Juge, Nucl. Phys. 106 (Proc. Suppl.) (2002) 847.

[61] S. Narison, Phys. Lett. B 520 (2001) 115.

[62] M. Jamin and B. Lange, Phys. Rev. D 65 (2002) 056005.

[63] J.H. Kühn and M. Steinhauser, Nucl. Phys. B 619 (2001) 588.

[64] J. Peñarrocha and K. Schilcher, Phys. Lett. B 515 (2001) 291.

[65] J. ERLER AND M. LuO, hep-ph/0207114.

[66] B.L. Ioffe And K.N. Zyablyuk, hep-ph/0207183.

[67] J.H. Kühn, A.A. Penin and A.A. Pivovarov, Nucl. Phys. B 534 (1998) 356.

[68] K. Melnikov and A. Yelkhovsky, Phys. Rev. D 59 (1999) 114009.

[69] M. Jamin and A. Pich, Nucl. Phys. 74 (Proc. Suppl.) (1999) 300. 
[70] M. Beneke And A. Signer, Phys. Lett. B 471 (1999) 233.

[71] A.H. Hoang, Phys. Rev. D 61 (2000) 034005.

[72] A.H. HoAng, hep-ph/0008102.

[73] A. Ali Khan et Al., Phys. Rev. D 62 (2000) 054505.

[74] V. Giménez, L. Giusti, G.Martinelli and F. Rapuano, JHeP 0003 (2000) 018.

[75] A.H. HoAng, hep-ph/0204299.

[76] S. Groote And A.A. Pivovarov, JETP Lett. 75 (2002) 221.

[77] S. Groote And A.A. Pivovarov, Eur. Phys. J. C 21 (2001) 133.

[78] M. Peter, Nucl. Phys. B 501 (1997) 471.

[79] Y. SchröDer, Phys. Lett. B 447 (1999) 321. 